\title{
Prostate Magnetic Resonance Imaging, with or Without Magnetic Resonance Imaging-targeted Biopsy, and Systematic Biopsy for Detecting Prostate Cancer: A Cochrane Systematic Review and Meta-analysis
}

\author{
Frank-Jan H. Drost ${ }^{a, b}$, Daniel Osses ${ }^{a, b}$, Daan Nieboer ${ }^{b, c}$, Chris H. Bangma ${ }^{b}$, \\ Ewout W. Steyerberg ${ }^{c}$, Monique J. Roobol ${ }^{b}$, Ivo G. Schoots ${ }^{a, *}$ \\ ${ }^{a}$ Department of Radiology \& Nuclear Medicine, Erasmus University Medical Center Rotterdam, Rotterdam, The Netherlands; ${ }^{\mathrm{b}}$ Department of Urology, \\ Erasmus University Medical Center Rotterdam, Rotterdam, The Netherlands; ${ }^{\mathrm{c}}$ Department of Public Health, Erasmus University Medical Center Rotterdam, \\ Rotterdam, The Netherlands
}

\section{Article info}

Article history:

Accepted June 19, 2019

Associate Editor:

James Catto

\section{Keywords:}

Prostate

Neoplasm

Biopsy

Magnetic resonance imaging

Diagnostic test accuracy

Systematic review

Meta-analysis

\begin{abstract}
Context: Magnetic resonance imaging (MRI), with or without MRI-targeted biopsy (MRI pathway), is an alternative test to systematic transrectal ultrasonography-guided biopsy in men suspected of having prostate cancer. At present, evidence on which test to use is insufficient to inform detailed evidence-based decision making.

objective: To determine the diagnostic accuracy of the index tests MRI only, MRItargeted biopsy, MRI pathway, and systematic biopsy, as compared with templateguided biopsy (reference standard), in detecting clinically significant prostate cancer, defined as International Society of Urological Pathology grade 2 or higher, in biopsynaive men or those with a prior-negative biopsy (or mix of both).

Evidence acquisition: We systematically searched the literature and considered for inclusion any cross-sectional study if it investigated (1) one or more index tests verified by the reference standard, and (2) paired testing of the MRI pathway with systematic biopsy. Quality and certainty of evidence were assessed by the Quality Assessment of Diagnostic Accuracy Studies (QUADAS-2) and Grading of Recommendations Assessment, Development and Evaluation, respectively.

Evidence synthesis: Accuracy analyses: Using a baseline cancer prevalence of $30 \%$, MRI pathway (sensitivity 0.72 [95\% confidence interval $\{\mathrm{CI}\}$ : $0.60-0.82]$; specificity 0.96 [0.94-0.98]; eight studies) may result in 216 (180-246) true positives, 28 (14-42) false positives, 672 (658-686) true negatives, and 84 (54-120) false negatives per 1000 men. Systematic biopsy (sensitivity 0.63 [0.19-0.93]; specificity 1.00 [0.91-1.00];

\footnotetext{
is This article is based on a Cochrane Review published. Drost FH, Osses DF, Nieboer D, Steyerberg EW, Bangma CH, Roobol MJ, et al. Prostate MRI, with or without MRI-targeted biopsy, and systematic biopsy for detecting prostate cancer. The Cochrane Database of Systematic Reviews 2019;4: Cd012663. https://www.cochranelibrary.com/cdsr/doi/10.1002/14651858.CD012663.pub2/full. Cochrane Reviews are regularly updated as new evidence emerges and in response to feedback, and the Cochrane Database of Systematic Reviews should be consulted for the most recent version of the review.

* Corresponding author. Department of Radiology \& Nuclear Medicine (Room Ns-549), Erasmus University Medical Centre, P.O. Box 2040, Rotterdam 3000 CA, The Netherlands; Dr. Molenwaterplein 40, 3015GD Rotterdam, The Netherlands. Tel. +31 107042006.

E-mail address: i.schoots@erasmusmc.nl (I.G. Schoots).
} 
four studies) may result in 189 (57-279) true positives, 0 (0-63) false positives, 700 (637-700) true negatives, and 111 (21-243) false negatives per 1000 men. Agreement analyses: With a direct comparison of the MRI pathway with systematic biopsy concerning significant disease, we found pooled detection ratios of 1.05 (95\% CI: 0.95-1.16; 20 studies) in biopsy-naive men and 1.44 (1.19-1.75; 10 studies) in men with a prior-negative biopsy. Concerning insignificant disease, we found detection ratios of 0.63 (95\% CI: $0.54-0.74$ ), and 0.62 (95\% CI: $0.44-0.88$ ), respectively.

Conclusions: MRI pathway had the most favourable outcome in significant and insignificant prostate cancer detection compared with systematic biopsy. The certainty in our findings was reduced by study limitations.

Patient summary: We reviewed recent advances in prostate biopsy by magnetic resonance imaging (MRI) guidance and targeting for prostate cancer detection in comparison with standard diagnosis by systematic biopsies. The findings of this Cochrane review suggest that MRI pathway is better than systematic biopsies in making a correct diagnosis of clinically important prostate cancer and reducing redundant biopsies and the detection of unimportant cancers substantially. However, MRI pathway still misses some men with important prostate cancer. Therefore, further research in this area is important.

(C) 2019 European Association of Urology. Published by Elsevier B.V. All rights reserved.

\section{Introduction}

To reduce overdiagnosis and overtreatment of indolent prostate cancer, while improving the detection of clinically significant prostate cancer and reducing the number of biopsy procedures, we need more accurate diagnostic methods and better risk stratification [1]. In a recent international multicentre randomised controlled trial, magnetic resonance imaging (MRI) in combination with MRI-targeted biopsy (MRI pathway) detected an absolute $12 \%$ more clinically significant prostate cancer and 13\% less indolent prostate cancer than systematic biopsy in biopsynaive men, and achieved $28 \%$ reduction of biopsies, because men with negative MRI did not receive prostate biopsy [2]. These results indicate that prebiopsy MRI and MRItargeted biopsy in the presence of an MRI-suspicious lesion would be superior to a systematic biopsy. If this is confirmed by other studies and longer follow-up of men who were not biopsied, it may initiate a change to guidelines.

Previous systematic reviews on diagnostic performances of the MRI pathway or prebiopsy MRI approach [3-11] have been based on study designs that did not accurately capture target conditions and index or reference test definitions, leading to a number of biases and inaccurate findings. Studies in these reviews included mainly men with positive MRI and disregarded men with negative MRI, inevitably leading to inaccurate true- and false-negative values of the MRI pathway. In addition, these reviews used systematic biopsy or radical whole-mount surgical specimens as reference standards, which inherently have a number of biases. Furthermore, the established definitions of clinically significant prostate cancer, based on histology from systematic biopsy and possibly additional nonhistological parameters, cannot be applied to results from the MRI pathway [12]. In this (copublished) Cochrane review and meta-analysis [13] we have largely overcome these limitations.

\section{Evidence acquisition}

For further detailed information on methods, we refer to the original Cochrane review [13].

\subsection{Objectives}

We aimed to determine the diagnostic accuracy of the index tests MRI only, MRI-targeted biopsy, MRI pathway (MRI with or without MRI-targeted biopsy), and systematic biopsy, as compared with template-guided biopsy as the reference standard, in detecting International Society of Urological Pathology (ISUP) grade 2 or higher (primary target condition), grade 3 or higher, and grade 1 prostate cancer (secondary target condition). Furthermore, we aimed to determine the agreement and disagreement, and the potential change in the number of biopsy procedures between the two index tests, MRI pathway, and systematic biopsy, for detecting the primary and secondary target conditions.

\subsection{Inclusion criteria}

\subsubsection{Types of studies}

We considered any cross-sectional study, if it investigated (Fig. 1) the following: (1) diagnostic test accuracy of one or more of the index tests (MRI, MRI pathway;including MRItargeted biopsy], or systematic biopsy) verified by the reference standard (template-guided biopsy), with each index test and reference standard performed in the same men or compared as in a randomised trial of test accuracy; or (2) agreement evidence between the MRI pathway and systematic biopsy, with each test performed in the same men.

Studies involving MRI had to report on both MRIpositive and MRI-negative men. The primary target condition had to be reported on a per-participant basis for all studies (Fig. 2). 


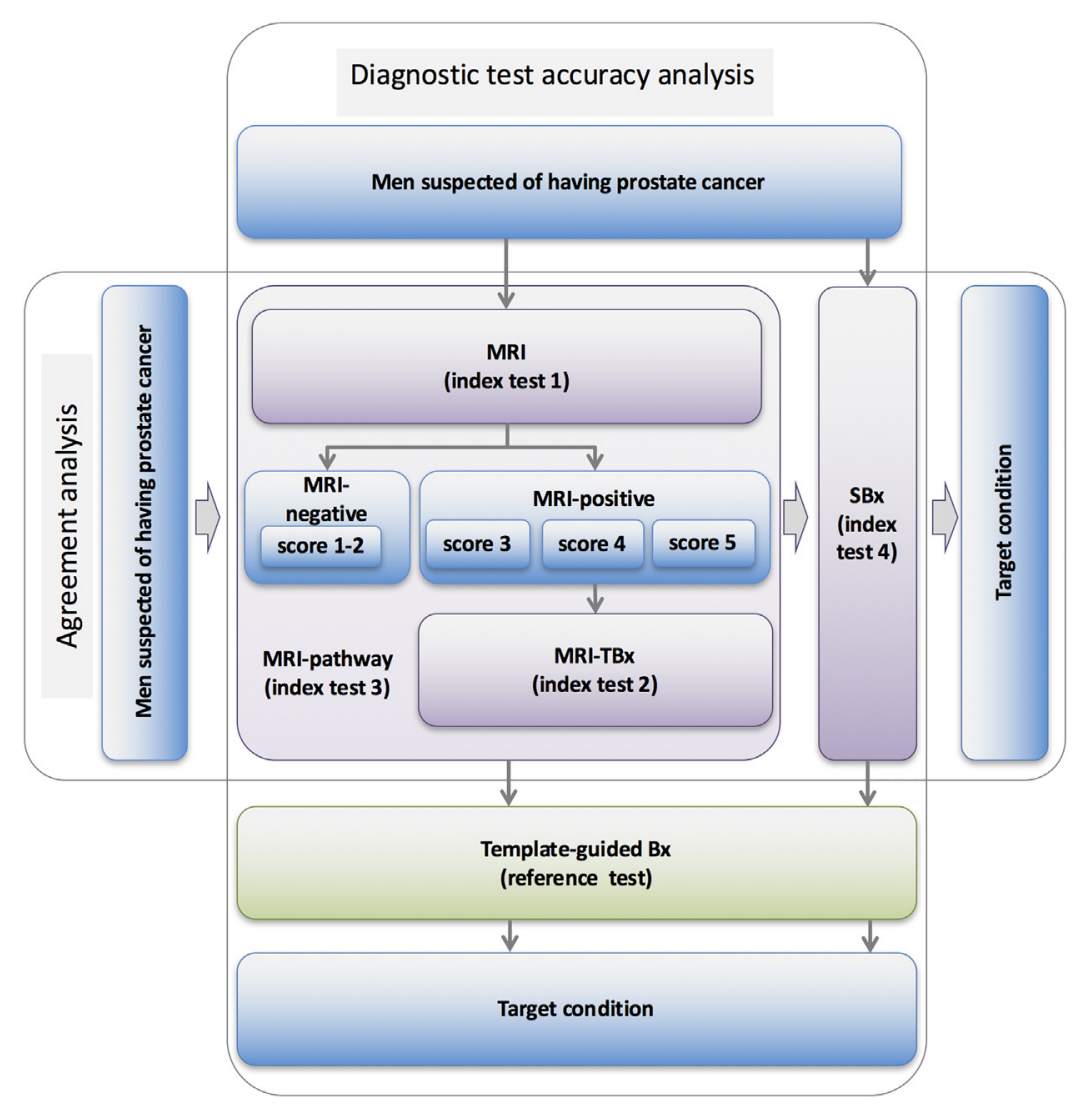

Fig. 1 - Clinical pathway flow diagram and study design.

\subsubsection{Study population}

The study population consisted of men with a clinical suspicion of prostate cancer (based on prostate-specific antigen or digital rectal examination outcome) in the biopsy-naive or prior-negative biopsy setting (or a mix of both).

\subsubsection{Index tests}

MRI (index test 1) comprised at least T2-weighted imaging and one functional imaging technique (diffusion-weighted imaging or dynamic contrast-enhanced imaging), reported according to any MRI-scoring system, mainly based on a five-point scale (Likert or Prostate Imaging Reporting and Data System) [14,15]. We defined the default threshold for MRI-positivity as 3/5 or more where possible. MRI-targeted biopsy (index test 2) included only MRI-positive men. The MRI pathway (index test 3 ) included MRI-positive men (in whom MRI-targeted biopsy was performed) and MRInegative men (in whom no MRI-targeted biopsy was performed). Systematic biopsy (index test 4) included either systematic transrectal or transperineal ultrasoundguided biopsies. We defined the MRI pathway and systematic biopsy as positive when histopathology of one of the target conditions in the biopsy cores was confirmed.

\subsubsection{Reference standard}

Template-guided biopsy, including transperineal templateguided mapping biopsy and the template-guided saturation biopsy, served as the reference standard $[16,17]$. We defined a positive template-guided biopsy as histopathological confirmation of one of the target conditions within the biopsy cores.

\subsubsection{Target conditions}

We solely focused on target conditions based on histological definitions according to the ISUP grading, as was recommended by International Working Group on Standards of Reporting for MRI-targeted biopsy studies (START) in order to overcome differences between definitions and biopsy methods [18]. The primary target condition was clinically significant prostate cancer, defined as ISUP grade 2 or higher based on histopathology findings, and scored as Gleason score (GS) $3+4$ or higher [19]. Secondary target conditions were grade 1 (GS $3+3$, indolent prostate cancer) and grade 3 or higher (GS $4+3$ or higher).

\subsection{Search strategy}

We performed a comprehensive search with no restriction on language or status of publication (including on-going studies), in electronic databases (CENTRAL, MEDLINE, Embase, and nine other databases), and updated to 31 July 2018 (Supplementary material, Appendix 1).

\subsection{Data collection and analysis}

2.4.1. Selection of studies, data extraction, and management Two reviewers independently screened all abstracts and full-text articles for eligibility, and extracted data using a 


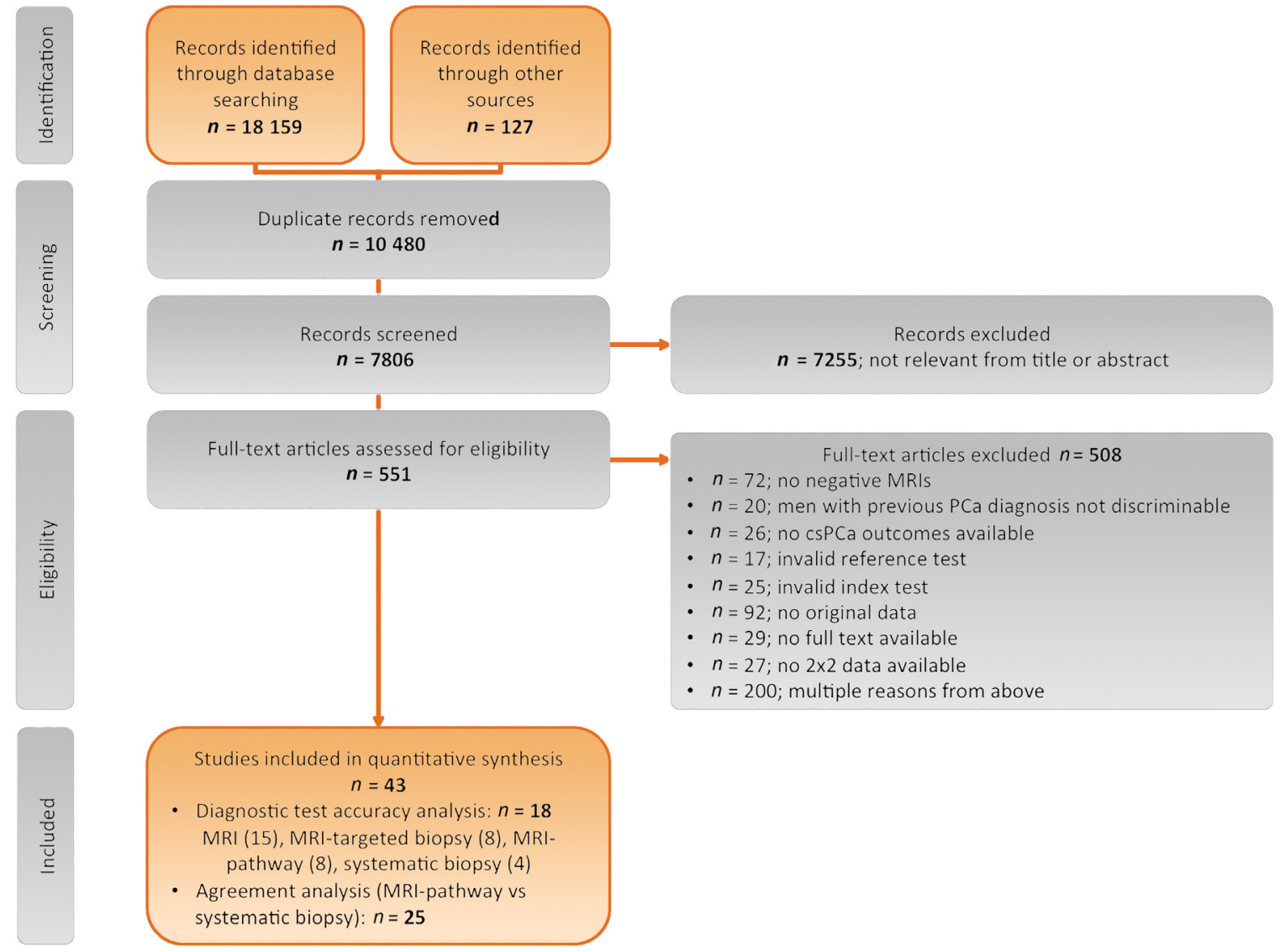

Fig. 2 - Study flowchart. csPCa = clinically significant prostate cancer; MRI = magnetic resonance imaging; MRI pathway = magnetic resonance imaging with subsequent magnetic resonance imaging-targeted biopsy; $\mathbf{P C a}=$ prostate cancer.

predefined data-extraction form. We constructed two-bytwo tables for cross-classification of the index tests versus reference standard for test accuracy data and the MRI pathway versus systematic biopsy for agreement data, based on per-participant data (Supplementary material, Appendix 2).

\subsubsection{Assessment of methodological quality}

Two reviewers independently assessed all included studies for methodological quality using the Quality Assessment of Diagnostic Accuracy Studies (QUADAS-2) tool [20], tailored to this Cochrane review [13].

\subsubsection{Statistical analysis and data synthesis}

For the test accuracy analysis, we calculated pooled estimates of sensitivity and specificity using the bivariate model [21]. For the agreement analysis (MRI pathway vs systematic biopsy), we calculated the proportion of detected cases (total number of cancers) as the number of concordant positive results plus the number of discordant positive results of both tests (Supplementary material, Appendix 2). We calculated the detection rate of either test as the number of positive results of that test divided by the total number of cancers detected. We synthesised pooled estimates of detection ratios (detection rate of MRI pathway/detection rate of systematic biopsy) by performing random-effect meta-analyses. We used mixed models (multinomial logistic regression models with a random intercept for study effects) to calculate pooled proportions of concordance and discordance between tests (Cochrane review [13]). Added value (discordance) data were constructed such that we assessed the tests as add-on tests (ie, considering reclassification by each test; Supplementary material, Appendix 3). We used Statistical Analysis Software (SAS), version 9.3, for Windows and $\mathrm{R}$ version 3.5 .0 to perform all statistical analyses.

2.4.4. Investigations of heterogeneity and sensitivity analyses To explore sources of heterogeneity, we assessed covariates by adding them one by one in our bivariate model: population setting, MRI magnet strength, MRI sequences, MRI-positivity threshold, endorectal coil, MRI-targeted biopsy method, biopsy approach, and radiologists' experience. We tested the same covariates using meta-regression techniques for the detection ratio in the agreement analysis.

\subsubsection{Certainty of evidence}

We rated the certainty of evidence on a per-outcome basis according to Grading of Recommendations Assessment, Development and Evaluation (GRADE) guidance for studies of diagnostic accuracy [22]. For the four main 
comparisons, we rated the certainty of evidence using GRADEpro GDT.

\section{Evidence synthesis}

For further detailed information on results, we refer to the original Cochrane review [13].

\subsection{Results of the search}

A total of 43 studies were eligible for inclusion in this review (Fig. 1) and provided data for multiple tests (Supplementary material, Appendix 4). Eighteen studies addressed the test accuracy analysis (index tests vs reference standard): 15 studies on MRI, eight studies on MRI, MRI-targeted biopsy, and MRI pathway in the same men and four studies on systematic biopsy (Table 1). These studies included 6871 men, of whom 5075 were biopsy naive, and 1796 had a history of at least one prior-negative biopsy. Twenty-five studies addressed the agreement analysis between MRI pathway and systematic biopsy in detecting prostate cancer with 6944 men, of whom 5353 were biopsy naive and 1591 had a history of at least one prior-negative biopsy (Table 1).

\subsection{Methodological quality of included studies}

As a result of QUADAS-2 assessment (Supplementary Fig. 2), we acknowledge overall concerns about the independence and applicability of tests in both test accuracy and agreement analyses, for which we performed sensitivity analyses to exclude studies with such quality concerns.

\subsection{Findings}

3.3.1. Test accuracy analysis (index tests verified by reference standard)

3.3.1.1. Detection of grade 2 or higher prostate cancer. MRI (pooled sensitivity of 0.91 [95\% confidence interval $\{\mathrm{CI}\}$ 0.83-0.95], specificity of 0.37 [0.29-0.46]; 12 studies, 3091 men; Table 2) at a baseline prevalence of 30\% (300/1000) may result in 273 (249-285) true positives, 441 (378-497) false positives, 259 (203-322) true negatives, and 27 (15-51) false negatives per 1000 men (Table 3). Hence, MRI did not identify 9\% (27/300) of men with grade 2 or higher prostate cancer.

These accuracy and predictive metrics are also presented for the index tests MRI-targeted biopsy, MRI pathway, and systematic biopsy (Tables 2 and 3). MRI-targeted biopsy, MRI pathway, and systematic biopsy missed, respectively, $20 \%$ (60/300), 28\% (84/300), and 37\% (111/300) of men with grade 2 or higher prostate cancer at the prevalence of $30 \%$ (300/1000), identified by the reference standard. Implications of these results, taking into account each step in the MRI pathway (MRI with subsequent MRI-targeted biopsy in MRI-positive men only) and systematic biopsy, are shown in Fig. 3.

A comparison of MRI with MRI pathway showed a substantial decrease in sensitivity (from 0.91 to 0.72 ; Fig. 4) and an increase in specificity (from 0.37 to 0.96 ), which were both statistically significant $(p<0.01$; Table 2$)$. Comparing MRI pathway with systematic biopsy showed a substantial decrease in sensitivity ( 0.72 vs $0.63 ; p=0.06$; Table 2) and similar specificities (Fig. 4).

At a baseline prevalence of $30 \%$ grade 2 or higher prostate cancer, the negative predictive values for MRI, MRI-targeted biopsy, MRI pathway, and systematic biopsy are 91\% (8694\%), 92 (88-94\%), 89\% (85-92\%), and 86\% (65-95\%), respectively (Table 2 ). Consequently, in the MRI pathway, negative MRI falsely predicts the absence of grade 2 or higher prostate cancer in $9 \%$ of men, while a negative systematic biopsy falsely predicts the absence of grade 2 or higher prostate cancer in $14 \%$ of men.

3.3.1.2. Detection of grade 1 prostate cancer. The pooled sensitivity and specificity for detecting grade 1 prostate cancer of all index tests are shown in Table 2. Comparing the sensitivity of the MRI pathway and systematic biopsy, the MRI pathway potentially avoided the detection of $66 \%$ of men with grade 1 prostate cancer, whereas systematic biopsy potentially avoided $45 \%$ of men with grade 1 prostate cancer $(p=0.52)$.

3.3.1.3. Detection of grade 2 or higher prostate cancer at a higher MRIpositive threshold. In clinical practice, lesions with an MRI suspicion score of 3 (likelihood for clinically significant cancer is equivocal [23]) might or might not be targeted with biopsies. By increasing the threshold of MRI positivity from $3 / 5$ to $4 / 5$, the proportion of negative MRI increased from $30 \%(23-38 \%)$ to $59 \%$ (43-74\%). The pooled sensitivity of MRI for detecting grade 2 or higher prostate cancer decreased from $0.89(0.82-0.94)$ to $0.72(0.52-0.86)$. The pooled specificity increased from $0.39(0.32-0.47)$ to 0.78 (0.68-0.86). Consequently, with a threshold 4/5 for MRI positivity, negative MRI missed identifying $28 \%$ of men with grade 2 or higher prostate cancer.

\subsubsection{Agreement analysis between MRI pathway and systematic biopsy}

In this section, we focused on agreement and disagreement (concordance and discordance) in the number of target conditions identified by the MRI pathway and systematic biopsy.

3.3.2.1. Detection of grade 2 or higher prostate cancer. In a mixed population (of biopsy-naive and prior-negative biopsy men), the pooled detection ratio of grade 2 or higher prostate cancer was 1.12 (1.02-1.23; 25 studies, 6944 men), meaning that the MRI pathway increased the detection rate of grade 2 or higher prostate cancer by $12 \%$ compared with systematic biopsy.

For men in the biopsy-naive setting, cancer proportion (total prostate cancer detected by both tests) was $27.7 \%$ (23.7-32.6\%; 20 studies, 5219 men) versus prior-negative biopsy setting of 22.8\% (20.0-26.2\%; 10 studies, 1564 men; Table 4$)$. The pooled detection ratios for grade 2 or higher prostate cancer were $1.05(0.95-1.16)$, and $1.44(1.19-1.75)$, respectively ( $p<0.01$; Fig. 5$)$. When focusing on only MRIpositive men in both subgroups, the pooled detection ratio 
Table 1 - Characteristic of the diagnostic test accuracy and agreement studies

\begin{tabular}{|c|c|c|c|c|c|c|c|c|c|c|c|c|c|c|}
\hline \multirow{3}{*}{$\begin{array}{l}\text { Study } \\
\text { Author } \\
\text { (year) }\end{array}$} & \multicolumn{6}{|c|}{ Tests } & \multirow{3}{*}{$\begin{array}{c}\text { Target } \\
\text { condition } \\
\text { ISUP } \\
\text { grade (G) }\end{array}$} & \multicolumn{3}{|c|}{ Recruitment } & \multicolumn{4}{|c|}{ Patient characteristics } \\
\hline & \multicolumn{4}{|c|}{ Index tests } & \multirow{2}{*}{$\begin{array}{l}\text { Reference } \\
\text { standard } \\
\text { Technique }\end{array}$} & \multirow[b]{2}{*}{ Independence } & & \multirow[b]{2}{*}{$\begin{array}{l}\text { Study } \\
\text { design }\end{array}$} & \multirow[b]{2}{*}{$\begin{array}{c}\text { Consecutive } \\
\text { enrolment }\end{array}$} & \multirow[b]{2}{*}{ Population } & \multirow[b]{2}{*}{$\begin{array}{c}\text { No. of } \\
\text { participants }\end{array}$} & \multirow[b]{2}{*}{$\begin{array}{l}\text { Median age } \\
\text { (range/SD) }\end{array}$} & \multirow[b]{2}{*}{$\begin{array}{l}\text { Median } \\
\text { PSA ng/ml } \\
\text { (range) }\end{array}$} & \multirow[b]{2}{*}{$\begin{array}{c}\text { Median } \\
\text { prostate } \\
\text { volume } \mathrm{cm}^{3} \\
\text { (range) }\end{array}$} \\
\hline & $\begin{array}{l}\text { Index tests } \\
\text { analysed }\end{array}$ & $\begin{array}{c}\text { MRI scale; } \\
\text { threshold }\end{array}$ & $\begin{array}{l}\text { MRI TBx, } \\
\text { technique }\end{array}$ & Route & & & & & & & & & & \\
\hline \multicolumn{15}{|c|}{ Diagnostic test accuracy studies } \\
\hline $\begin{array}{l}\text { Abd-Alazeez } \\
(2014)\end{array}$ & MRI & $1-5 ; \geq 3$ & Cognitive & Transperineal & TTMB & No & $\begin{array}{l}G=1, \geq 1, \\
\geq 2, \geq 3\end{array}$ & Retrospective & No & $\begin{array}{l}\text { Prior- } \\
\text { negative } \mathrm{Bx}\end{array}$ & 54 & $\begin{array}{l}64 \\
(39-75)\end{array}$ & $\begin{array}{l}10 \\
(2-23)\end{array}$ & $\begin{array}{l}53 \\
(19-136)\end{array}$ \\
\hline $\begin{array}{l}\text { Ahmed } \\
(2017)\end{array}$ & MRI, SBx & $1-5 ; \geq 3$ & NA & Transrectal & TTMB & Yes & $\begin{array}{l}\mathrm{G}=1, \geq 1, \\
\geq 2, \geq 3\end{array}$ & Prospective & Yes & Bx naive & 576 & $63(7.6)^{b}$ & $7.1(2.9)^{\mathrm{b}}$ & $\mathrm{NR}$ \\
\hline $\begin{array}{l}\text { Dal Moro } \\
\text { (2019) }\end{array}$ & $\begin{array}{l}\text { MRI, } \\
\text { MRI TBx, } \\
\text { MRI pathway }\end{array}$ & $1-5 ; \geq 3$ & Cognitive & Transrectal & $\mathrm{TSB}^{\mathrm{c}}$ & Yes & $\begin{array}{l}G=1, \geq 1 \\
\geq 2, \geq 3\end{array}$ & Prospective & Yes & $\begin{array}{l}\text { Prior- } \\
\text { negative Bx }\end{array}$ & 123 & $\begin{array}{l}62 \\
\left(57-68^{\mathrm{d}}\right)\end{array}$ & $\begin{array}{l}6.3 \\
\left(4.8-8.9^{\mathrm{d}}\right)\end{array}$ & $\begin{array}{l}55 \\
(20-149)^{\mathrm{b}}\end{array}$ \\
\hline $\begin{array}{l}\text { Distler } \\
(2017)\end{array}$ & $\begin{array}{l}\text { MRI, MRI TBx, } \\
\text { MRI pathway }\end{array}$ & $1-5 ; \geq 3$ & Software & Transperineal & $\mathrm{TSB}^{\mathrm{e}}$ & No & $G \geq 2$ & Prospective & Yes & Mixed $^{f}$ & $\begin{array}{l}1040 \\
(597 / 443)\end{array}$ & $\begin{array}{l}65 \\
\left(60-71^{\mathrm{d}}\right)\end{array}$ & $\begin{array}{l}7.2 \\
\left(5.3-10.4^{\mathrm{d}}\right)\end{array}$ & $\begin{array}{l}45 \\
\left(34-64^{\mathrm{d}}\right)\end{array}$ \\
\hline \multirow[t]{2}{*}{$\begin{array}{l}\text { Grey } \\
(2015)\end{array}$} & MRI & $1-5 ; \geq 3$ & Cognitive & Transperineal & $\mathrm{TSB}^{\mathrm{e}}$ & No & $\begin{array}{l}G=1, \geq 1, \\
\geq 2, \geq 3\end{array}$ & Prospective & Yes & Mixed $^{f}$ & 83 & $64(6.8)^{b}$ & $\begin{array}{l}13.3 \\
(12.1)^{\mathrm{b}}\end{array}$ & $68(35)^{b}$ \\
\hline & & & & & & & & & & & 103 & $65(7.6)^{a}$ & $\begin{array}{l}12.6 \\
(13.7)^{\mathrm{a}}\end{array}$ & $54(31)^{a}$ \\
\hline $\begin{array}{l}\text { Hansen } \\
(2016)\end{array}$ & $\begin{array}{l}\text { MRI, MRI TBx, } \\
\text { MRI pathway }\end{array}$ & $1-5 ; \geq 3$ & $\begin{array}{l}\text { Software/ } \\
\text { transperineal }\end{array}$ & Transperineal & $\mathrm{TSB}^{\mathrm{e}}$ & Unclear & $\begin{array}{l}G=1, \geq 1, \\
\geq 2, \geq 3\end{array}$ & Prospective & Yes & $\begin{array}{l}\text { Prior- } \\
\text { negative Bx }\end{array}$ & 295 & $\begin{array}{l}65 \\
\left(59-69^{d}\right)\end{array}$ & $\begin{array}{l}7.8 \\
\left(6.0-12^{\mathrm{d}}\right)\end{array}$ & $\begin{array}{l}65 \\
\left(44-83^{d}\right)\end{array}$ \\
\hline \multirow[t]{2}{*}{$\begin{array}{l}\text { Hansen } \\
(2018)\end{array}$} & MRI & $1-5 ; \geq 3$ & Software & Transperineal & $\mathrm{TSB}^{\mathrm{e}}$ & No & $\begin{array}{l}G=1, \geq 1, \\
\geq 2, \geq 3\end{array}$ & Prospective & Yes & $\begin{array}{l}\text { Bx naive } \\
\text { (centre 1) }\end{array}$ & 163 & $\begin{array}{l}64 \\
\left(57-69^{d}\right)\end{array}$ & $\begin{array}{l}6.6 \\
\left(4.6-9.0^{\mathrm{d}}\right)\end{array}$ & $\begin{array}{l}44 \\
\left(33-55^{\mathrm{d}}\right)\end{array}$ \\
\hline & & & Cognitive & & & & & & & $\begin{array}{l}\text { Bx naive } \\
\text { (centre 3) }\end{array}$ & 242 & $\begin{array}{l}65 \\
\left(60-70^{d}\right)\end{array}$ & $\begin{array}{l}5.9 \\
\left(4.6-8.0^{\mathrm{d}}\right)\end{array}$ & $\begin{array}{l}25 \\
\left(24-47^{\mathrm{d}}\right)\end{array}$ \\
\hline $\begin{array}{l}\text { Hansen } \\
(2017)\end{array}$ & $\begin{array}{l}\text { MRI, MRI TBx, } \\
\text { MRI pathway }\end{array}$ & $1-5 ; \geq 3$ & $\begin{array}{l}\text { Software/ } \\
\text { transperineal }\end{array}$ & Transperineal & $\mathrm{TSB}^{\mathrm{e}}$ & Unclear & $G \geq 2$ & Prospective & Unclear & $\begin{array}{l}\text { Prior- } \\
\text { negative Bx }\end{array}$ & 287 & $\begin{array}{l}66 \\
\left(61-72^{\mathrm{d}}\right)\end{array}$ & $\begin{array}{l}9.7 \\
\left(7.1-13.9^{d}\right)\end{array}$ & $\begin{array}{l}52 \\
\left(36-75^{\mathrm{d}}\right)\end{array}$ \\
\hline $\begin{array}{l}\text { Kesch } \\
\text { (2017) }\end{array}$ & $\begin{array}{l}\text { MRI, MRI TBx, } \\
\text { MRI pathway }\end{array}$ & $1-5 ; \geq 3$ & $\begin{array}{l}\text { Software/ } \\
\text { transperineal }\end{array}$ & Transperineal & $\mathrm{TSB}^{\mathrm{g}}$ & Yes & $\begin{array}{l}G=1, \geq 1, \\
\geq 2, \geq 3\end{array}$ & Prospective & Unclear & Mixed $^{f}$ & $\begin{array}{l}146 \\
(95 / 51)\end{array}$ & $\begin{array}{l}65 \\
\left(58-71^{\mathrm{d}}\right)\end{array}$ & $\begin{array}{l}7.2 \\
\left(5.4-10.2^{\mathrm{d}}\right)\end{array}$ & $\begin{array}{l}46 \\
\left(36-60^{\mathrm{d}}\right)\end{array}$ \\
\hline $\begin{array}{l}\text { Lawrence } \\
\text { (2014) }\end{array}$ & $\begin{array}{l}\text { MRI, MRI TBx, } \\
\text { MRI pathway }\end{array}$ & $1-4 ; \geq 2$ & Software & Transperineal & $\mathrm{TSB}^{\mathrm{e}}$ & No & $\begin{array}{l}G=1 \\
\geq 1, \geq 2\end{array}$ & Retrospective & No & $\begin{array}{l}\text { Prior- } \\
\text { negative Bx }\end{array}$ & 39 & $\begin{array}{l}64 \\
(47-77)^{\mathrm{b}}\end{array}$ & $\begin{array}{l}10 \\
(1.2-36)\end{array}$ & $\mathrm{NR}$ \\
\hline \multirow[t]{2}{*}{$\begin{array}{l}\text { Mortezavi } \\
\text { (2018) }\end{array}$} & $\begin{array}{l}\text { MRI, MRI TBx, } \\
\text { MRI pathway }\end{array}$ & $1-5 ; \geq 3$ & Software & Transrectal & TSB & No & $\begin{array}{l}G=1, \geq 1, \\
\geq 2, \geq 3\end{array}$ & Retrospective & Yes & Bx naive & 163 & $\begin{array}{l}63 \\
\left(57-68^{\mathrm{d}}\right)\end{array}$ & $\begin{array}{l}5.8 \\
\left(4.4-8.9^{\mathrm{d}}\right)\end{array}$ & $\begin{array}{l}44 \\
\left(34-60^{\mathrm{d}}\right)\end{array}$ \\
\hline & & & & & & & & & & $\begin{array}{l}\text { Prior- } \\
\text { negative Bx }\end{array}$ & 86 & $\begin{array}{l}64 \\
\left(60-69^{\mathrm{d}}\right)\end{array}$ & $\begin{array}{l}8.6 \\
\left(5.7-13^{\mathrm{d}}\right)\end{array}$ & $\begin{array}{l}54 \\
\left(41-70^{\mathrm{d}}\right)\end{array}$ \\
\hline \multirow[t]{2}{*}{$\begin{array}{l}\text { Muthuveloe } \\
\text { (2016) }\end{array}$} & MRI & $1-5 ; \geq 3$ & NA & NA & $\mathrm{TSB}^{\mathrm{h}}$ & Unclear & $\begin{array}{l}G=1, \geq 1, \\
\geq 2, \geq 3\end{array}$ & Retrospective & Unclear & Bx naive & 9 & $\begin{array}{l}68 \\
(46-81)\end{array}$ & $\begin{array}{l}11.5 \\
(1.2-92.5)\end{array}$ & NR \\
\hline & & & & & & & & & & $\begin{array}{l}\text { Prior- } \\
\text { negative Bx }\end{array}$ & 162 & $\begin{array}{l}65 \\
(47-78)\end{array}$ & $\begin{array}{l}10 \\
(2.7-61)\end{array}$ & NR \\
\hline $\begin{array}{l}\text { Pepe } \\
\text { (2013) }\end{array}$ & $\begin{array}{l}\text { MRI, MRI TBx, } \\
\text { MRI pathway }\end{array}$ & $0-1: \geq 1$ & Cognitive & Transrectal & $\mathrm{TSB}^{\mathrm{h}}$ & No & $\begin{array}{l}G=1, \\
\geq 1, \geq 2\end{array}$ & Prospective & Unclear & $\begin{array}{l}\text { Prior- } \\
\text { negative Bx }\end{array}$ & 78 & $63(49-72)$ & $11(3.7-45)$ & NR \\
\hline $\begin{array}{l}\text { Thompson } \\
\text { (2016) }\end{array}$ & MRI & $1-5 ; \geq 3$ & $\begin{array}{l}\text { Software, } \\
\text { cognitive }\end{array}$ & Transperineal & TTMB & No & $\begin{array}{l}G=1, \geq 1 \\
\geq 2, \geq 3\end{array}$ & Prospective & Yes & Bx naive & 344 & $63\left(56-67^{d}\right)$ & $\begin{array}{l}5.2 \\
\left(3.7-7.1^{\mathrm{d}}\right)\end{array}$ & $\begin{array}{l}40 \\
\left(30-54^{\mathrm{d}}\right)\end{array}$ \\
\hline $\begin{array}{l}\text { Tsivian } \\
\text { (2017) }\end{array}$ & MRI & $1-5 ; \geq 3$ & NA & NA & TTMB & Yes & $\begin{array}{l}G=1, \geq 1, \\
\geq 2, \geq 3\end{array}$ & Retrospective & Unclear & $\begin{array}{l}\text { Prior- } \\
\text { negative Bx }\end{array}$ & 33 & $65\left(61-69^{\mathrm{d}}\right)$ & $\begin{array}{l}7.1(5.1- \\
\left.13.6^{\mathrm{d}}\right)\end{array}$ & $\begin{array}{l}44 \\
\left(32-65^{\mathrm{d}}\right)\end{array}$ \\
\hline $\begin{array}{l}\text { Nafie } \\
\text { (2014) }\end{array}$ & SBx & NA & NA & Transrectal & $\mathrm{TSB}^{\mathrm{h}}$ & Yes & $\begin{array}{l}G=1, \geq 1, \\
\geq 2, \geq 3\end{array}$ & Prospective & Unclear & Bx naive & 50 & $67(54-84)^{\mathrm{b}}$ & $8(4-18)^{b}$ & $\begin{array}{l}58 \\
(19-165)^{\mathrm{b}}\end{array}$ \\
\hline
\end{tabular}


Table 1 (Continued)

\begin{tabular}{|c|c|c|c|c|c|c|c|c|c|c|c|c|c|c|}
\hline \multirow{3}{*}{$\begin{array}{l}\text { Study } \\
\text { Author } \\
\text { (year) }\end{array}$} & \multicolumn{6}{|c|}{ Tests } & \multirow{3}{*}{$\begin{array}{c}\text { Target } \\
\text { condition } \\
\\
\text { ISUP } \\
\text { grade (G) }\end{array}$} & \multicolumn{3}{|c|}{ Recruitment } & \multicolumn{4}{|c|}{ Patient characteristics } \\
\hline & \multicolumn{4}{|c|}{ Index tests } & \multirow{2}{*}{$\begin{array}{l}\text { Reference } \\
\text { standard } \\
\text { Technique }\end{array}$} & \multirow[b]{2}{*}{ Independence } & & \multirow[b]{2}{*}{$\begin{array}{l}\text { Study } \\
\text { design }^{\text {a }}\end{array}$} & \multirow[b]{2}{*}{$\begin{array}{l}\text { Consecutive } \\
\text { enrolment }\end{array}$} & \multirow[b]{2}{*}{ Population } & \multirow[b]{2}{*}{$\begin{array}{c}\text { No. of } \\
\text { participants }\end{array}$} & \multirow[b]{2}{*}{$\begin{array}{l}\text { Median age } \\
\text { (range/SD) }\end{array}$} & \multirow[b]{2}{*}{$\begin{array}{l}\text { Median } \\
\text { PSA ng/ml } \\
\text { (range) }\end{array}$} & \multirow[b]{2}{*}{$\begin{array}{c}\text { Median } \\
\text { prostate } \\
\text { volume } \mathrm{cm}^{3} \\
\text { (range) }\end{array}$} \\
\hline & $\begin{array}{l}\text { Index tests } \\
\text { analysed }\end{array}$ & $\begin{array}{l}\text { MRI scale; } \\
\text { threshold }\end{array}$ & $\begin{array}{l}\text { MRI TBx, } \\
\text { technique }\end{array}$ & Route & & & & & & & & & & \\
\hline Nafie (2017) & SBX & NA & $\mathrm{NA}$ & Transrectal & $\mathrm{TSB}^{\mathrm{h}}$ & Yes & $G=1, \geq 1, \geq 2$ & Prospective & Unclear & $\begin{array}{l}\text { Prior- } \\
\text { negative Bx }\end{array}$ & 42 & $65(50-75)^{\mathrm{b}}$ & $\begin{array}{l}8.3(4.4- \\
19)^{\mathrm{b}}\end{array}$ & $59(21-152)^{b}$ \\
\hline $\begin{array}{l}\text { Ploussard } \\
\text { (2014) }\end{array}$ & SBx & NA & NA & Transrectal & $\mathrm{TSB}^{\mathrm{C}}$ & No & $G=1, \geq 1, \geq 2$ & Prospective & Yes & Bx naive & 2753 & $64(8)^{b}$ & $12.5(7.2)^{\mathrm{b}}$ & $46(25)^{b}$ \\
\hline \multicolumn{15}{|c|}{ Agreement studies } \\
\hline \multirow[t]{2}{*}{$\begin{array}{l}\text { Alberts } \\
(2017)\end{array}$} & $\begin{array}{l}\text { MRI pathway } \\
\text { vs SBx }\end{array}$ & $1-5 ; \geq 3$ & Software & Transrectal & NA & Yes & $\begin{array}{l}G=1, \geq 1, \geq 2, \\
\geq 3\end{array}$ & Prospective & Yes & Bx naive & 74 & $73\left(72-74^{\mathrm{d}}\right)$ & $\begin{array}{l}4.2(3.4- \\
\left.5.8^{\mathrm{d}}\right)\end{array}$ & $53\left(37-71^{\mathrm{d}}\right)$ \\
\hline & & & & & & & & & & $\begin{array}{l}\text { Prior- } \\
\text { negative Bx }\end{array}$ & 84 & & & \\
\hline $\begin{array}{l}\text { Boesen } \\
(2017)\end{array}$ & $\begin{array}{l}\text { MRI pathway } \\
\text { vs SBx }\end{array}$ & $1-5 ; \geq 3$ & Software & Transrectal & NA & Yes & $\begin{array}{l}G=1, \geq 1, \geq 2, \\
\geq 3\end{array}$ & Prospective & Unclear & $\begin{array}{l}\text { Prior- } \\
\text { negative } \mathrm{Bx}\end{array}$ & 206 & $65\left(58-68^{d}\right)$ & $\begin{array}{l}12.8(8.9- \\
\left.19.6^{\mathrm{d}}\right)\end{array}$ & NR \\
\hline $\begin{array}{l}\text { Boesen } \\
(2018)\end{array}$ & $\begin{array}{l}\text { MRI pathway } \\
\text { vs SBx }\end{array}$ & $1-5 ; \geq 3$ & Software & Transrectal & NA & Yes & $\begin{array}{l}\bar{G}=1, \geq 1, \geq 2, \\
\geq 3\end{array}$ & Prospective & Yes & Bx naive & 1020 & $67\left(61-71^{\mathrm{d}}\right)$ & $8\left(5.7-13^{\mathrm{d}}\right)$ & $53\left(40-72^{\mathrm{d}}\right)$ \\
\hline $\begin{array}{l}\text { Castellucci } \\
\text { (2017) }\end{array}$ & $\begin{array}{l}\text { MRI pathway } \\
\text { vs SBx }\end{array}$ & $1-5 ; \geq 3$ & Cognitive & Transrectal & NA & Unclear & $\begin{array}{l}G=1, \geq 1, \geq 2, \\
\geq 3\end{array}$ & Prospective & Yes & Bx naive & 168 & $61(8)^{\mathrm{f}}$ & $8.3(6.1)^{\mathrm{f}}$ & $49(7)^{\mathrm{f}}$ \\
\hline $\begin{array}{l}\text { Chang } \\
(2017)\end{array}$ & $\begin{array}{l}\text { MRI pathway } \\
\text { vs SBx }\end{array}$ & $1-5 ; \geq 3$ & Cognitive & Transrectal & NA & No & $\begin{array}{l}G=1, \geq 1, \geq 2, \\
\geq 3\end{array}$ & Retrospective & Yes & $\begin{array}{l}\text { Prior- } \\
\text { negative Bx }\end{array}$ & 65 & $64\left(60-68^{d}\right)$ & $\begin{array}{l}10.9(7.2- \\
\left.14.7^{\mathrm{d}}\right)\end{array}$ & $48\left(34-63^{\mathrm{d}}\right)$ \\
\hline Chen (2015) & $\begin{array}{l}\text { MRI pathway } \\
\text { vs SBx }\end{array}$ & $1-5 ; \geq 3$ & Cognitive & Transperineal & $\mathrm{NA}$ & Yes & $\bar{G} \geq 2$ & Prospective & Yes & Bx naive & 420 & $67(45-91)$ & $\begin{array}{l}9.7(2.4- \\
35.7)\end{array}$ & $45(21-83)$ \\
\hline \multirow[t]{2}{*}{ Cool (2016) } & $\begin{array}{l}\text { MRI pathway } \\
\text { vs SBx }\end{array}$ & Other & Software & Transrectal & NA & Unclear & $G=1, \geq 1, \geq 2$ & Prospective & Unclear & Bx naive & 50 & $59(8)^{\mathrm{f}}$ & $6.0(3.5)^{\mathrm{f}}$ & $38(18)^{\mathrm{f}}$ \\
\hline & & & & & & & & & & $\begin{array}{l}\text { Prior- } \\
\text { negative Bx }\end{array}$ & 50 & $62(7)^{\mathrm{f}}$ & $7.9(3.9)^{f}$ & $56(27)^{\mathrm{f}}$ \\
\hline Costa (2013) & $\begin{array}{l}\text { MRI pathway } \\
\text { vs SBx }\end{array}$ & $1-5 ; \geq 4$ & Cognitive & Transrectal & NA & No & $\mathrm{G} \geq 2, \geq 3$ & Retrospective & No & $\begin{array}{l}\text { Prior- } \\
\text { negative Bx }\end{array}$ & 38 & $64(48-77)^{f}$ & $\begin{array}{l}14.4(1.8- \\
33.1)^{\mathrm{f}}\end{array}$ & NR \\
\hline $\begin{array}{l}\text { Delong- } \\
\text { champs } \\
\text { (2013) }\end{array}$ & $\begin{array}{l}\text { MRI pathway } \\
\text { vs SBx }\end{array}$ & $1-5 ; \geq 3$ & Software & Transrectal & $\mathrm{NA}$ & Unclear & $G \geq 2$ & Prospective & Yes & Bx naive & 391 & $64(7)^{\mathrm{f}}$ & $8.5(3.9)^{f}$ & $56(30)^{\mathrm{f}}$ \\
\hline \multirow[t]{2}{*}{ Filson (2016) } & $\begin{array}{l}\text { MRI pathway } \\
\text { vs SBx }\end{array}$ & $1-5 ; \geq 3$ & Software & Transrectal & NA & Unclear & $G \geq 2, \geq 3$ & Prospective & Yes & Bx naive & 329 & $64\left(59-69^{d}\right)$ & $\begin{array}{l}5.8(4.4- \\
\left.8.1^{\mathrm{d}}\right)\end{array}$ & $45\left(33-62^{d}\right)$ \\
\hline & & & & & & & & & & $\begin{array}{l}\text { Prior- } \\
\text { negative Bx }\end{array}$ & 324 & $66\left(59-70^{d}\right)$ & $\begin{array}{l}7.6(5- \\
\left.11.5^{\mathrm{d}}\right)\end{array}$ & $58\left(40-84^{\mathrm{d}}\right)$ \\
\hline $\begin{array}{l}\text { Garcia } \\
\text { Bennett } \\
\text { (2017) }\end{array}$ & $\begin{array}{l}\text { MRI pathway } \\
\text { vs SBx }\end{array}$ & $1-5 ; \geq 3$ & Cognitive & Transperineal & $\mathrm{NA}$ & Yes & $\begin{array}{l}G=1, \geq 1, \geq 2, \\
\geq 3\end{array}$ & Prospective & Unclear & Bx naive & 60 & $64(6.7)^{f}$ & $7.2\left(6-9.4^{\mathrm{d}}\right)$ & $48\left(35-63^{\mathrm{d}}\right)$ \\
\hline \multirow[t]{2}{*}{$\begin{array}{l}\text { Grönberg } \\
\text { (2018) }\end{array}$} & $\begin{array}{l}\text { MRI pathway } \\
\text { vs SBx }\end{array}$ & $1-5 ; \geq 3$ & Software & Transrectal & NA & No & $\begin{array}{l}G=1, \geq 1, \geq 2, \\
\geq 3\end{array}$ & Prospective & Yes & Bx naive & 387 & $64(45-74)^{\mathrm{f}}$ & $6.3\left(4.4^{\mathrm{d}}\right)$ & $(32-70)^{i}$ \\
\hline & & & & & & & & & & $\begin{array}{l}\text { Prior- } \\
\text { negative Bx }\end{array}$ & 145 & & & \\
\hline $\begin{array}{l}\text { Jambor } \\
(2015)\end{array}$ & $\begin{array}{l}\text { MRI pathway } \\
\text { vs SBx }\end{array}$ & $1-5 ; \geq 4$ & Cognitive & Transrectal & NA & Yes & $\begin{array}{l}G=1, \geq 1, \geq 2, \\
\geq 3\end{array}$ & $\begin{array}{l}\text { Unclear, } \\
\text { unclear }\end{array}$ & Unclear & Bx naive & 53 & $66(47-76)$ & $7.4(4-14)$ & $42(17-107)$ \\
\hline $\begin{array}{l}\text { Jambor } \\
\text { (2017) }\end{array}$ & $\begin{array}{l}\text { MRI pathway } \\
\text { vs SBx }\end{array}$ & $1-5 ; \geq 3$ & Cognitive & Transrectal & $\mathrm{NA}$ & No & $\begin{array}{l}\bar{G}=1, \geq 1, \geq 2, \\
\geq 3\end{array}$ & Prospective & Unclear & Mixed & 134 & $65(6)^{f}$ & $\begin{array}{l}7.5(5.7- \\
\left.9.6^{\mathrm{d}}\right)\end{array}$ & $37\left(28-49^{\mathrm{d}}\right)$ \\
\hline
\end{tabular}


Table 1 (Continued)

\begin{tabular}{|c|c|c|c|c|c|c|c|c|c|c|c|c|c|c|}
\hline \multirow[t]{2}{*}{ Study } & \multicolumn{6}{|c|}{ Tests } & \multirow{2}{*}{$\begin{array}{c}\text { Target } \\
\text { condition }\end{array}$} & \multicolumn{3}{|c|}{ Recruitment } & \multicolumn{4}{|c|}{ Patient characteristics } \\
\hline & \multicolumn{4}{|c|}{ Index tests } & \multirow{2}{*}{\multicolumn{2}{|c|}{$\begin{array}{l}\text { Reference } \\
\text { standard } \\
\text { Technique }\end{array}$}} & & \multirow[b]{2}{*}{$\begin{array}{l}\text { Study } \\
\text { design }^{\mathrm{a}}\end{array}$} & \multirow[b]{2}{*}{$\begin{array}{l}\text { Consecutive } \\
\text { enrolment }\end{array}$} & \multirow[b]{2}{*}{ Population } & \multirow[b]{2}{*}{$\begin{array}{c}\text { No. of } \\
\text { participants }\end{array}$} & \multirow[b]{2}{*}{$\begin{array}{l}\text { Median age } \\
\text { (range/SD) }\end{array}$} & \multirow[b]{2}{*}{$\begin{array}{l}\text { Median } \\
\text { PSA ng/ml } \\
\text { (range) }\end{array}$} & \multirow[b]{2}{*}{$\begin{array}{l}\text { Median } \\
\text { prostate } \\
\text { volume } \mathrm{cm}^{3} \\
\text { (range) }\end{array}$} \\
\hline $\begin{array}{l}\text { Author } \\
\text { (year) }\end{array}$ & $\begin{array}{c}\text { Index tests } \\
\text { analysed }\end{array}$ & $\begin{array}{l}\text { MRI scale; } \\
\text { threshold }\end{array}$ & $\begin{array}{l}\text { MRI TBx, } \\
\text { technique }\end{array}$ & Route & & & $\begin{array}{c}\text { ISUP } \\
\text { grade }(G)\end{array}$ & & & & & & & \\
\hline
\end{tabular}

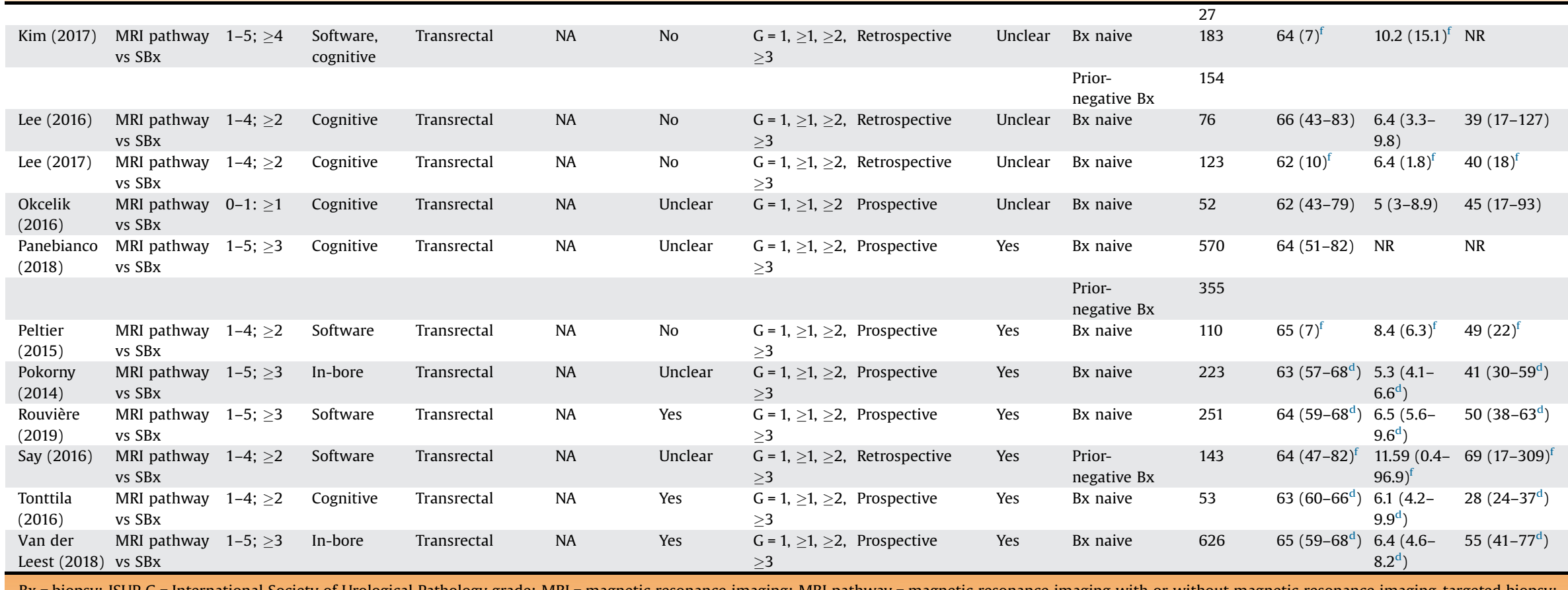

$\mathrm{Bx}=$ biopsy; ISUP $\mathrm{G}=$ International Society of Urological Pathology grade; $\mathrm{MRI}=$ magnetic resonance imaging; MRI pathway = magnetic resonance imaging with or without magnetic resonance imaging-targeted biopsy; MRI TBX = magnetic resonance imaging-targeted biopsy; $N=$ number; $N A=$ not applicable; $N R=$ not reported; $P S A=$ prostate-specific antigen; $S B x=$ systematic biopsy; $S D=$ standard deviation; TSB $=$ transperineal

saturation biopsy; TTMB = transperineal template mapping biopsy.

a Included participants were part of the same study cohort (no randomised populations were included).

b Included participants were part of the same study cohort (no randomised populations were included).

c Transrectal.

d Interquartile range (as opposed to range).

e Ginsburg biopsies.

f Mean value (as opposed to median value).

${ }^{g}$ Transperineal optimised prostate biopsy.

${ }^{\mathrm{h}}$ In-house transperineal saturation biopsy.

i Range of interquartile ranges across three centres. 
Table 2 - Diagnostic accuracy and predictive metrics of the index tests verified by template-guided biopsy as the reference standard for different target conditions

\begin{tabular}{|c|c|c|c|c|c|c|c|c|c|c|}
\hline \multirow[t]{2}{*}{ Target condition } & \multirow[t]{2}{*}{ Index test $^{\mathrm{a}}$} & \multirow{2}{*}{$\begin{array}{l}\text { No. of participants } \\
\text { (studies) }\end{array}$} & \multirow{2}{*}{$\begin{array}{l}\text { Proportion } \\
\text { negative MRI } \\
(95 \% \mathrm{CI})\end{array}$} & \multicolumn{3}{|c|}{ Accuracy metrics } & \multirow{2}{*}{$\begin{array}{l}\text { Prevalence }^{\mathrm{b}} \\
(95 \% \mathrm{CI})\end{array}$} & \multirow{2}{*}{$\begin{array}{l}\text { Assumptive } \\
\text { prevalence }^{c}\end{array}$} & \multicolumn{2}{|c|}{ Predictive metrics } \\
\hline & & & & $\begin{array}{l}\text { Sensitivity } \\
(95 \% \mathrm{CI})\end{array}$ & $\begin{array}{l}\text { Specificity } \\
(95 \% \mathrm{CI})\end{array}$ & $p$ value & & & $\mathrm{NPV}^{\mathrm{d}}(95 \% \mathrm{CI})$ & $\operatorname{PPV}^{\mathrm{d}}(95 \% \mathrm{CI})$ \\
\hline \multirow[t]{4}{*}{ ISUP $G \geq 2$ prostate cancer } & MRI & $3091(12)$ & $0.29(0.2$ & $0.91(0.83-0.95)$ & $0.37(0.29-0.46)$ & $p<0.01^{\mathrm{e}}$ & $0.29(0.22-0.38)$ & 0.30 & $0.91(0.86-0.94)$ & $0.38(0.36-0.40)$ \\
\hline & MRI Tbx ${ }^{f}$ & $1553(8)$ & NA & $0.80(0.69-0.87)$ & $0.94(0.90-0.97)$ & & $0.34(0.24-0.46)$ & & $0.92(0.88-0.94)$ & $0.85(0.77-0.91)$ \\
\hline & MRI pathway & $2257(8)$ & $0.29(0.24-0.35)$ & $0.72(0.60-0.82)$ & $0.96(0.94-0.98)$ & $p=0.06^{g}$ & $0.26(0.18-0.36)$ & & $0.89(0.85-0.92)$ & $0.90(0.83-0.94)$ \\
\hline & SBx & $3421(4)$ & NA & $0.63(0.19-0.93)$ & $1.00(0.91-1.00)$ & & $0.34(0.21-0.51)$ & & $0.86(0.65-0.95)$ & $1.00(0.73-1.00)$ \\
\hline \multirow[t]{4}{*}{ ISUP $G \geq 3$ prostate cancer } & MRI & $1438(7)$ & $0.31(0.21-0.42)$ & $0.95(0.87-0.99)$ & $0.35(0.26-0.46)$ & $\mathrm{ID}^{\mathrm{e}}$ & $0.14(0.08-0.23)$ & 0.15 & $0.98(0.95-0.99)$ & $0.21(0.19-0.23)$ \\
\hline & MRI Tbx ${ }^{f}$ & $428(3)$ & NA & ID & ID & & $0.21(0.12-0.35)$ & & ID & ID \\
\hline & MRI pathway & $604(3)$ & $0.29(0.26-0.33)$ & ID & ID & $\mathrm{ID}^{\mathrm{g}}$ & $0.16(0.09-0.27)$ & & ID & ID \\
\hline & SBx & $626(2)$ & NA & ID & ID & & ID & & ID & ID \\
\hline \multirow[t]{4}{*}{ ISUP $\mathrm{G}=1$ prostate cancer } & MRI & $1764(10)$ & $0.28(0.20-0.38)$ & $0.70(0.59-0.80)$ & $0.27(0.19-0.37)$ & $p<0.01^{\mathrm{e}}$ & $0.20(0.17-0.23)$ & 0.20 & $0.79(0.74-0.82)$ & $0.20(0.18-0.21)$ \\
\hline & MRI Tbx ${ }^{f}$ & $497(5)$ & NA & $0.51(0.21-0.81)$ & $1.00(0.77-1.00)$ & & $0.22(0.19-0.26)$ & & $0.89(0.80-0.94)$ & $0.97(0.21-1.00)$ \\
\hline & MRI pathway & $681(5)$ & $0.24(0.16-0.36)$ & $0.34(0.19-0.53)$ & $1.00(0.90-1.00)$ & $p=0.52^{g}$ & $0.21(0.18-0.24)$ & & $0.86(0.82-0.89)$ & $0.95(0.37-1.00)$ \\
\hline & SBX & $3421(4)$ & $\mathrm{NA}$ & $0.55(0.25-0.83)$ & $0.99(0.81-1.00)$ & & $0.20(0.16-0.25)$ & & $0.90(0.81-0.95)$ & $0.94(0.37-1.00)$ \\
\hline \multicolumn{11}{|c|}{$\begin{array}{l}\mathrm{CI}=\text { confidence interval; ISUP G = International Society of Urological Pathology grade; ID = inadequate data; MRI = magnetic resonance imaging; MRI pathway = magnetic resonance imaging with or without magnetic } \\
\text { resonance imaging-targeted biopsy; MRI TBx = magnetic resonance imaging-targeted biopsy; NA = not applicable; NPV = negative predictive value; PPV = positive predictive value; SBX = systematic biopsy. } \\
\text { a Data did not allow differentiation between the mix of included participants (biopsy-naive and prior-negative biopsy men). } \\
\text { b Prevalence is pooled estimate of all detected cancer by template-guided biopsy. } \\
\text { c Assumptive prevalence is an extrapolation from the pooled estimates of all detected cancer by template-guided biopsy per target condition. This assumptive prevalence is necessary for adequate comparison of PPVs and } \\
\text { NPVs between index tests. } \\
\text { d Based on the Bayes' theorem using the point estimates and } 95 \% \text { confidence intervals of the pooled positive and negative likelihood ratio and the point estimate of the prevalence. } \\
\text { e Comparing sensitivity between MRI and the MRI pathway. } \\
\text { f MRI-positive men only, instead of MRI-positive + MRI-negative men, implicating a higher risk profile and increased prevalence of clinically significant prostate cancer. } \\
\text { g Comparing sensitivity between the MRI pathway and SBx. }\end{array}$} \\
\hline
\end{tabular}

${ }^{f}$ MRI-positive men only, instead of MRI-positive + MRI-negative

Comparing sensitivity between the MRI pathway and SBx. 
Table 3 - Summary of Grading of Recommendations Assessment, Development and Evaluation (GRADE) guidance for diagnostic test accuracy of individual index tests

\begin{tabular}{|c|c|c|c|c|}
\hline Population: & \multicolumn{4}{|c|}{$\begin{array}{l}\text { Men suspected of having clinically significant prostate cancer undergoing their first biopsy (biopsy-naive men) or a repeat } \\
\text { biopsy (prior-negative biopsy men) }\end{array}$} \\
\hline Setting: & \multicolumn{4}{|c|}{ University hospitals and specialised care centres } \\
\hline $\begin{array}{l}\text { Reference } \\
\text { test: }\end{array}$ & \multicolumn{4}{|c|}{ Template-guided biopsy, which comprehensively samples all zones of the prostate } \\
\hline Threshold: & \multicolumn{4}{|c|}{ ISUP grade $\geq 2$ prostate cancer } \\
\hline Index test: & MRI & MRI-targeted biopsy & MRI pathway & Systematic biopsy \\
\hline Threshold: & MRI score $\geq 3$ out of 5 & ISUP grade $\geq 2$ prostate cancer & ISUP grade $\geq 2$ prostate cancer & ISUP grade $\geq 2$ prostate cancer \\
\hline Population: & $3091(12)$ & $1553(8)$ & $2257(8)$ & $3421(4)$ \\
\hline Pooled sensitivity: & 0.91 (95\% CI: $0.83-0.95)$ & 0.80 (95\% CI: 0.69-0.87) & 0.72 (95\% CI: $0.60-0.82)$ & 0.63 (95\% CI: 0.19-0.93) \\
\hline Pooled specificity: & 0.37 (95\% CI: 0.29-0.46) & 0.94 (95\% Cl: 0.90-0.97) & 0.96 (95\% CI: 0.94-0.98) & 1.00 (95\% CI: 0.91-1.00) \\
\hline \multicolumn{5}{|c|}{ Results per 1000 men tested $(95 \% \mathrm{CI})$ : at a baseline prevalence of $30 \%$ ISUP grade $\geq 2$ prostate cancer by the reference test } \\
\hline True positives: & $273(249-285)$ & $240(207-261)$ & $216(180-246)$ & $189(57-279)$ \\
\hline False negatives: & $27(15-51)$ & $60(39-93)$ & $84(54-120)$ & $111(21-243)$ \\
\hline True negatives: & $259(203-322)$ & $658(630-679)$ & $672(658-686)$ & $700(637-700)$ \\
\hline False positives: & $441(378-497)$ & $42(21-70)$ & $28(14-42)$ & $0(0-63)$ \\
\hline Certainty of evidence (tp/fn): & $\bullet \circ$ Low $^{\mathrm{a}, \mathrm{b}}$ & $0 \circ \operatorname{Low}^{\mathrm{a}, \mathrm{b}}$ & $0 \circ$ Low $^{\mathrm{a}, \mathrm{b}}$ & $\circ$ Moderate $^{\mathrm{a}, \mathrm{b}, \mathrm{c}}$ \\
\hline Certainty of evidence ( $\mathrm{tn} / \mathrm{fp})$ : & ○० Low ${ }^{\mathrm{a}, \mathrm{b}}$ & $\bigcirc \circ \operatorname{Low}^{\mathrm{a}, \mathrm{b}}$ & $0 \circ \operatorname{Low}^{\mathrm{a}, \mathrm{b}}$ & ○०० $\operatorname{Low}^{\mathrm{a}, \mathrm{b}, \mathrm{c}}$ \\
\hline \multicolumn{5}{|c|}{$\begin{array}{l}\mathrm{CI}=\text { confidence interval; fn = false negative-test indicates that clinically significant prostate cancer is not present but patient actually has clinically significant } \\
\text { prostate cancer; fp = false positive-test indicates clinically significant prostate cancer but patient actually does not have clinically significant prostate cancer; } \\
\text { ISUP = International Society of Urological Pathology; MRI = magnetic resonance imaging; tn = true negative-test indicates that clinically significant prostate } \\
\text { cancer is not present and patient actually does not have clinically significant prostate cancer; tp = true positive-indicates clinically significant prostate cancer } \\
\text { and patient actually has clinically significant prostate cancer. } \\
\text { a A considerable number of studies had a high or unclear risk of bias, mainly in the participant selection and reference standard domains. } \\
\text { b Considerable, clinically relevant, heterogeneity was observed across pooled study results. } \\
\text { c Important imprecision was noted, which contributed to decision to downgrade for inconsistency. }\end{array}$} \\
\hline
\end{tabular}

increased from 1.05 to $1.12(1.01-1.23)$ and from 1.44 to 1.49 (1.22-1.82), respectively.

3.3.2.2. Detection ratios for grade 1 prostate cancer. For men in the biopsy-naive and the prior-negative biopsy settings, cancer proportions of grade 1 prostate cancer were $27.2 \%$ (23.9$31.1 \%$; 17 studies, 4079 men) and 23.0\% (18.0-30.2\%; eight studies, 1202 men), respectively; the pooled detection ratio was $0.63(0.54-0.74)$ and $0.62(0.44-0.88)$, respectively (Table 4).

3.3.2.3. Added values (discordance) in detection of grade 2 or higher prostate cancer. Per 100 biopsy-naive men, the MRI pathway detected approximately 23 men with grade 2 or higher prostate cancer (23.4\% [19.4-28.2]; Table 4). In addition to the MRI pathway, systematic biopsy detected four additional men $(4.3 \%$ [2.6-6.9\%]). The total number of detected cases was $27(27.7 \%[23.7-32.6 \%])$. Conversely, systematic biopsy detected 21 men $(21.4 \%$ [17.2-26.5\%]) and the MRIpathway detected six additional men (6.3\% [4.8-8.2\%]). Further details on mixed population and prior-negative biopsy men are shown in the Cochrane review [13].

3.3.2.4. Added values (discordance) in detection of grade 1 prostate cancer. Per 100 biopsy-naive men, the MRI pathway detected approximately 11 men with grade 1 prostate cancer (11.2\% [8.4-14.9\%]; Table 4). In addition to the MRI pathway, systematic biopsy detected 10 additional men (9.8\% [8.0-11.8\%]). The total number of detected cases was 21 (20.9\% [18.0-24.7\%]). Conversely, systematic biopsy detected 19 men (18.5\% [15.6-22.2\%]) and the MRI pathway detected two additional men (2.4\% [1.4-4.0\%]).
3.3.2.5. Added values (discordance) in detection of grade 2 or higher prostate cancer in MRI-positive and MRI-negative men. Stratifying men further into having positive or negative MRI aids in interpreting the added value in each of these categories. The pooled proportions of positive and negative MRI were respectively $67.0 \%$ (58.7-74.4\%) and $33.0 \%$ (25.6-41.3\%) in the biopsy-naive setting, and were equivalent in the priornegative biopsy setting (Table 4).

Per 100 biopsy-naive men with positive MRI, the MRI pathway detected approximately 39 men with grade 2 or higher prostate cancer (39.2\% [33.3-45.7\%]). In addition to the MRI pathway, systematic biopsy detected five men $(4.9 \%$ [2.8-8.3\%]). The total number of detected cases was 44 (44.2\% [38.6-50.4\%]). Conversely, systematic biopsy detected 34 men (34.4\% [28.3-41.3\%]) and the MRI pathway detected 10 additional men (9.8\% [7.1-13.2\%]).

Per 100 biopsy-naive men with negative MRI, systematic biopsy detected eight additional men with grade 2 or higher prostate cancer (8.1\% [5.6-11.6\%]) and 18 additional men with grade 1 prostate cancer (18.4\% [14.2-23.7\%]).

\subsection{Heterogeneity analyses and sensitivity analyses}

For the test accuracy analyses (index tests vs reference standard [template-guided biopsy]), we observed considerable heterogeneity in all index tests (Cochrane review [13]). For the agreement analyses (MRI pathway vs systematic biopsy), the heterogeneity (total tausquare $=0.03$ ) is illustrated in Figure 5. We found a statistically significant difference in the detection ratio of the MRI pathway versus systematic biopsy between the subgroups of population (prior-negative biopsy vs biopsy 
a) Hypothetical cohort MRI result $\quad$ MRI-targeted Biopsy resulta
With an assumed prevalence of $30 \%$ prostate cancer

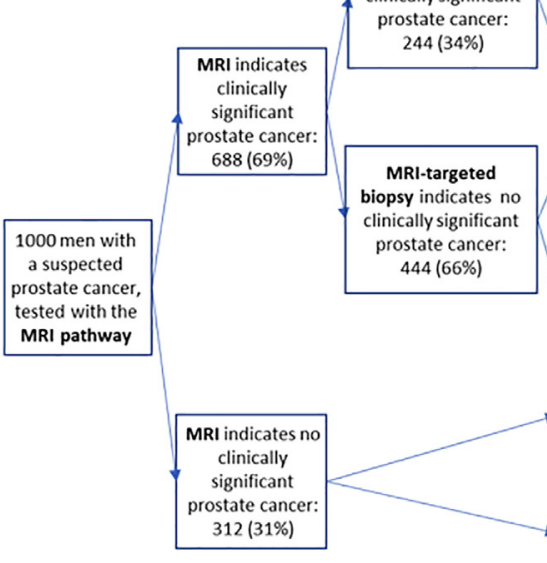

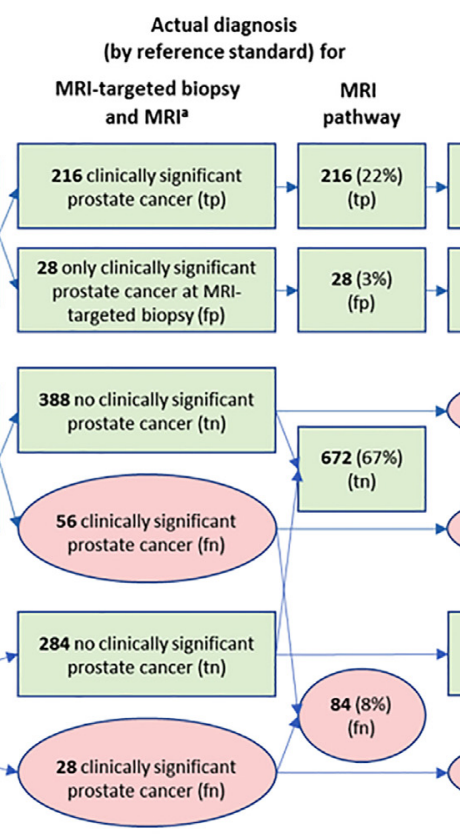

by reference standard) for

MRI

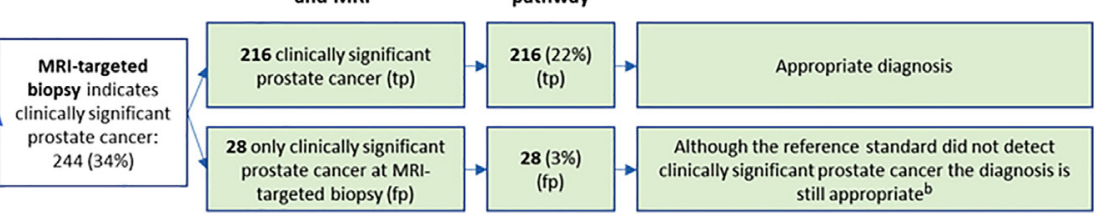

Potential unnecessarily induced patient burden

Potential unnecessarily induced patient burden,

clinically significant prostate cancer

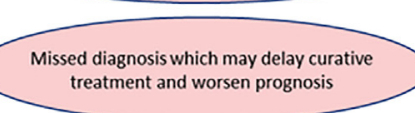

No MRI-targeted biopsy (appropriate)

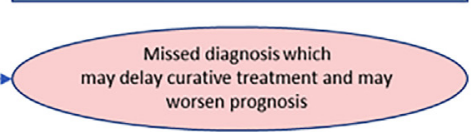

b)

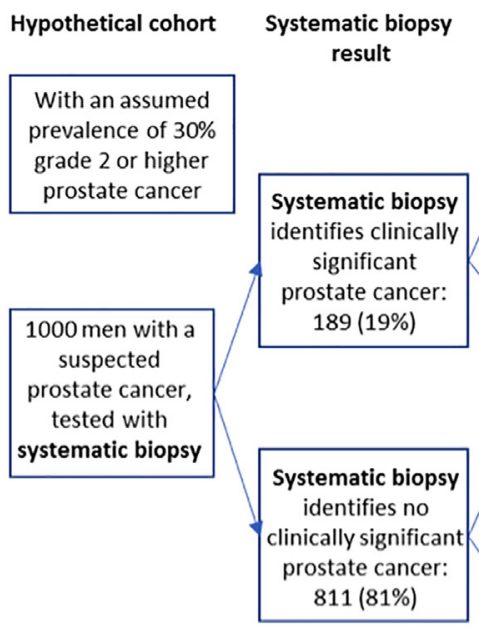

Actual diagnosis (by reference standard)
Implications

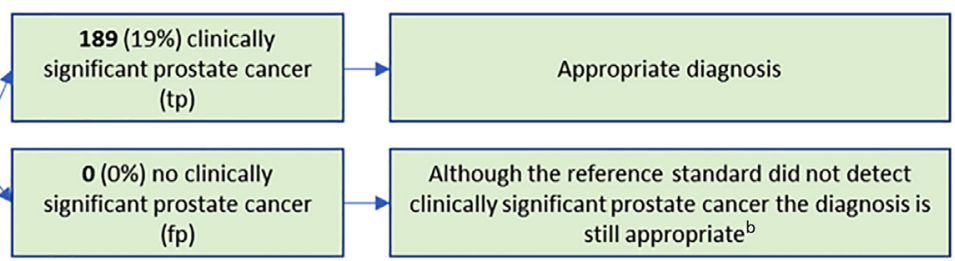
still appropriate ${ }^{b}$

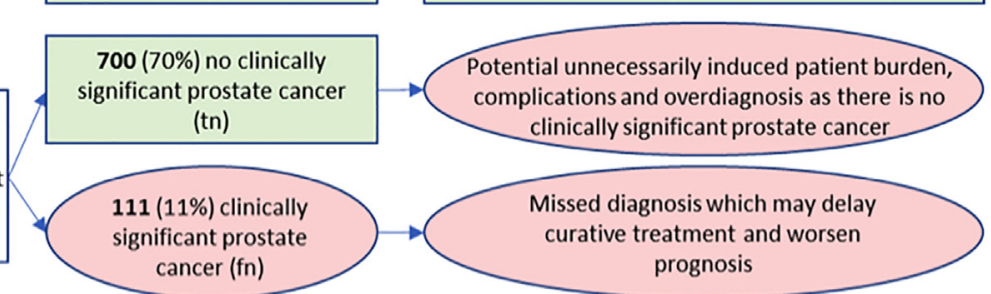

Fig. 3 - Test results and implications of a hypothetical cohort of 1000 men tested for prostate cancer using the (A) MRI pathway and (B) systematic biopsy. $\mathrm{fn}=$ false negative-test indicates that clinically significant prostate cancer is not present but patient actually has clinically significant prostate cancer; $f p=$ false positive-test indicates clinically significant prostate cancer but patient actually does not have clinically significant prostate cancer; MRI = magnetic resonance imaging; $t$ = true negative-test indicates that clinically significant prostate cancer is not present and patient actually does not have clinically significant prostate cancer; $t p=$ true positive-indicates clinically significant prostate cancer and patient actually has clinically significant prostate cancer. ${ }^{a}$ The numbers in this figure are based on findings of the MRI pathway; therefore, MRI and MRI-targeted biopsy results differ slightly from the numbers in Table 3 $^{\text {b }}$ Diagnoses by the MRI pathway and reference standard are based on biopsy histopathology, with equal chance of up- or downgrading following radical prostatectomy.

naive), suggesting that they may be sources of heterogeneity (Cochrane review [13]).

We performed sensitivity analyses for the detection of grade 2 or higher prostate cancer by excluding studies based on certain quality and additional criteria. Excluding studies with a high or an unclear risk of bias or applicability concern in one of the four QUADAS-2 domains did not substantially change the accuracy results of MRI, MRI-targeted biopsy, and the MRI pathway (Cochrane review [13]).

\subsection{Discussion}

This copublished Cochrane review presents the test accuracy of prostate MRI, MRI-targeted biopsy, MRI pathway (MRI with or without MRI-targeted biopsy), and current standard testing with systematic biopsies in prostate cancer diagnosis, using template-guided biopsy sampling of the whole prostate as the reference standard. This analysis provides evidence to determine their discriminative value in current clinical practice. Both the MRI 


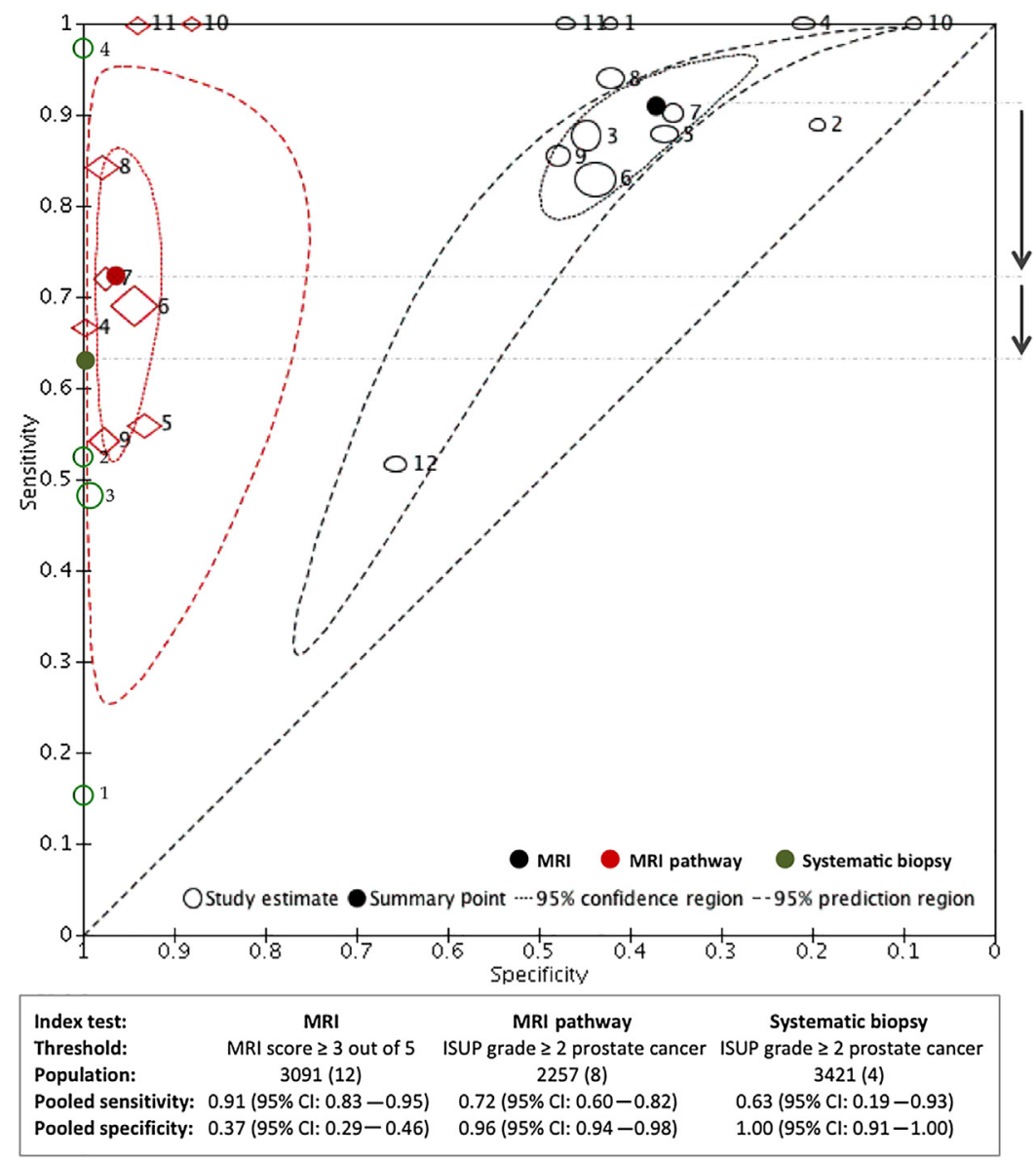

Fig. 4 - Comparison of diagnostic test accuracy between MRI, MRI pathway, and systematic biopsy for detecting ISUP grade 2 and higher prostate cancer. Summary ROC plots of MRI, MRI pathway, and systematic biopsy, verified by template-guided biopsy, with references to included studies (see original review for further details [1]). A comparison of MRI with MRI pathway showed a substantial decrease in sensitivity (from 0.91 to 0.72 ) and an increase in specificity (from 0.37 to 0.96 ), both of which were statistically significant $(p<0.01$; Table 3 ). A comparison of the MRI pathway with systematic biopsy showed a substantial decrease in sensitivity (from 0.72 to $0.63 ; p=0.06$; Table 3 ), and similar specificities. CI = confidence interval; ISUP = International Society of Urological Pathology; MRI = magnetic resonance imaging; ROC = receiver operating characteristics.

pathway and the systematic biopsy missed considerable proportions of grade 2 or higher prostate cancer, but the MRI pathway missed less than the systematic biopsy.

Furthermore, the agreement analyses for detecting prostate cancer between two index tests (the MRI pathway and the current practice of systematic biopsy) provide additional evidence for biopsy decision making, indicating that the MRI pathway is more favourable than systematic biopsy. The difference between the detection rates of the MRI pathway and systematic biopsy was largest in men with a prior-negative biopsy and insignificant in biopsy-naive men. Evidence further suggested that the MRI pathway beneficially missed more grade 1 prostate cancer than systematic biopsy in both population types. Therefore, the MRI pathway could potentially reduce the amount of overdiagnosis, and harms related to surveillance and overtreatment.

\subsubsection{MRI-directed biopsy management}

The benefits of using MRI (reducing biopsy procedures and the overdiagnosis of grade 1 prostate cancer with improving the detection of grade 2 and higher prostate cancer) are largest if MRI has a direct impact on biopsy decision management and shared decision making. In other words, the MRI before any biopsy and the MRI pathway as the replacement for systematic biopsy, thus omitting systematic biopsy in specified circumstances, might provide the most favourable diagnostic strategy.

Approximately one-third of all men had negative MRI. This is a substantially large population in whom additional systematic biopsies may potentially be avoided. Some expert centres even report up to 50\% MRI-negative men, suggesting that an even larger population may benefit when experience in MRI reading may improve [24]. The added value of performing systematic biopsy in MRI-negative men for the detection of grade 2 or higher prostate cancer could be considered as limited with regard to total detection and additional harms. As a prostate biopsy is associated with patient burden, infection, morbidity, overdiagnosis, and related overtreatment, it should be avoided when possible. Omitting systematic biopsy in men with negative MRI might be considered acceptable in some clinical situations. However, benefits and harms are difficult to balance on an individual basis. Therefore, men with negative MRI could 


\begin{tabular}{|c|c|c|c|c|c|c|c|c|c|c|c|c|}
\hline \multicolumn{2}{|c|}{ Population } & \multirow{2}{*}{$\begin{array}{l}\text { Target } \\
\text { condition } \\
\text { (ISUP } \\
\text { grade) }\end{array}$} & \multirow[t]{2}{*}{$\begin{array}{l}\text { Patients } \\
\text { (studies) }\end{array}$} & \multicolumn{6}{|c|}{$\begin{array}{l}\text { Proportion prostate cancer detected in \% } \\
\qquad(95 \% \mathrm{CI})\end{array}$} & \multicolumn{2}{|c|}{$\begin{array}{l}\text { Detection ratio } \\
(95 \% \mathrm{CI})^{\mathrm{b}}\end{array}$} & \multirow{2}{*}{$\begin{array}{c}\text { Difference } \\
\text { between } \\
\text { populations, } \\
p \text { value }^{c}\end{array}$} \\
\hline $\begin{array}{l}\text { Biopsy } \\
\text { status }\end{array}$ & $\begin{array}{l}\text { MRI in \% } \\
(95 \% \mathrm{CI})^{\mathrm{a}}\end{array}$ & & & $\begin{array}{c}\text { Combined MRI } \\
\text { pathway + SBx } \\
\text { (total cancer } \\
\text { detected) }\end{array}$ & $\begin{array}{c}\text { MRI } \\
\text { pathway }\end{array}$ & SBx & $\begin{array}{l}\text { Both MRI } \\
\text { pathway } \\
\text { and SBx }\end{array}$ & $\begin{array}{c}\text { Only by MRI } \\
\text { pathway } \\
\text { (added value) }\end{array}$ & $\begin{array}{l}\text { Only by SBx } \\
\text { (added value) }\end{array}$ & $\begin{array}{l}\text { MRI pathway } \\
\text { versus SBx }\end{array}$ & $p$ value & \\
\hline \multirow[t]{14}{*}{$\begin{array}{l}\text { Biopsy- } \\
\text { naive men }\end{array}$} & $\begin{array}{l}\text { Positive + negative } \\
(100[100-100])\end{array}$ & $G=1$ & 4079 (17) & NA & $13.5(10.7-17.2)$ & $\begin{array}{l}22.4 \\
(19.1-26.3)\end{array}$ & NA & $\mathrm{NA}$ & NA & $\begin{array}{l}0.630 \\
(0.535-0.742)\end{array}$ & 0.000 & 0.905 \\
\hline & & $G=1^{d}$ & 4079 (17) & $20.9(18.0-24.7)$ & $11.2(8.4-14.9)$ & $18.5(15.6-22.2)$ & $8.8(6.2-12.3)$ & $2.4(1.4-4.0)$ & $\begin{array}{l}9.8 \\
(8.0-11.8)\end{array}$ & $\begin{array}{l}0.611 \\
(0.485-0.769)\end{array}$ & 0.000 & - \\
\hline & & $G \geq 1$ & 4799 (19) & $53.2(48.7-57.9)$ & $41.0(35.8-46.4)$ & $47.8(42.8-52.9)$ & $35.6(30.2-41.2)$ & $5.4(3.6-8.0)$ & $\begin{array}{l}12.2 \\
(8.7-16.7)\end{array}$ & $\begin{array}{l}0.845 \\
(0.767-0.930)\end{array}$ & 0.001 & 0.121 \\
\hline & & $G \geq 2$ & $5219(20)$ & $27.7(23.7-32.6)$ & $23.4(19.3-28.1)$ & $21.4(17.2-26.5)$ & $17.1(13.0-22)$ & $6.3(4.8-8.2)$ & $\begin{array}{l}4.3 \\
(2.6-6.9)\end{array}$ & $\begin{array}{l}1.050 \\
(0.948-1.162)\end{array}$ & 0.349 & 0.002 \\
\hline & & $G \geq 3$ & $4306(16)$ & $15.5(12.6-19.5)$ & $12.7(9.9-16.5)$ & $10.8(8.0-14.8)$ & $8.0(5.4-11.6)$ & $4.7(3.5-6.3)$ & $\begin{array}{l}2.8 \\
(1.7-4.8)\end{array}$ & $\begin{array}{l}1.087 \\
(0.937-1.261)\end{array}$ & 0.269 & 0.004 \\
\hline & $\begin{array}{l}\text { Positive } \\
(67.0[58.7-74.4])\end{array}$ & $G=1$ & $2682(16)$ & NA & $21.3(17.0-26.9)$ & $23.7(19.6-29.1)$ & NA & NA & NA & $\begin{array}{l}0.854 \\
(0.743-0.982)\end{array}$ & 0.026 & 0.347 \\
\hline & & $G=1^{d}$ & $2682(16)$ & $21.1(16.7-27.1)$ & $17.0(12.6-22.9)$ & $17.7(13.3-23.8)$ & $13.6(9.3-19.5)$ & $3.4(2.1-5.3)$ & $\begin{array}{l}4.1 \\
(2.5-6.7)\end{array}$ & $\begin{array}{l}0.909 \\
(0.770-1.072)\end{array}$ & 0.257 & - \\
\hline & & $G \geq 1$ & 2955 (17) & $70.9(65.0-76.6)$ & $63.7(56.3-70.6)$ & $63.8(56.2-70.7)$ & $56.6(47.7-64.6)$ & $7.1(4.2-11.9)$ & $\begin{array}{l}7.2 \\
(4.7-10.8)\end{array}$ & $\begin{array}{l}0.994 \\
(0.915-1.079)\end{array}$ & 0.881 & 0.053 \\
\hline & & $G \geq 2$ & 2955 (17) & $44.2(38.6-50.4)$ & $39.2(33.3-45.7)$ & $34.4(28.3-41.3)$ & $29.5(23.2-36.5)$ & $9.8(7.1-13.2)$ & $\begin{array}{l}4.9 \\
(2.8-8.3)\end{array}$ & $\begin{array}{l}1.119 \\
(1.014-1.234)\end{array}$ & 0.025 & 0.005 \\
\hline & & $G \geq 3$ & 2899 (15) & $24.8(21.0-29.6)$ & $21.2(17.4-25.7)$ & $17.5(13.8-22.3)$ & $13.9(10.3-18.3)$ & $7.3(5.4-9.7)$ & $\begin{array}{l}3.7 \\
(2.2-6.1)\end{array}$ & $\begin{array}{l}1.158 \\
(1.024-1.310)\end{array}$ & 0.020 & 0.007 \\
\hline & $\begin{array}{l}\text { Negative } \\
(33.0[25.6-41.3])\end{array}$ & $G=1$ & $1287(16)$ & $18.4(14.2-23.7)$ & NA & $18.4(14.2-23.7)$ & NA & NA & $\begin{array}{l}18.4 \\
(14.2-23.7)\end{array}$ & NA & NA & NA \\
\hline & & $G \geq 1$ & 1343 (17) & $25.5(20.7-30.9)$ & NA & $25.5(20.7-30.9)$ & NA & NA & $\begin{array}{l}25.5 \\
(20.7-30.9)\end{array}$ & NA & $\mathrm{NA}$ & NA \\
\hline & & $G \geq 2$ & $1343(17)$ & $8.1(5.6-11.6)$ & NA & $8.1(5.6-11.6)$ & $\mathrm{NA}$ & $\mathrm{NA}$ & $\begin{array}{l}8.1 \\
(5.6-11.6)\end{array}$ & NA & NA & NA \\
\hline & & $G \geq 3$ & $1297(15)$ & $3.0(1.6-5.5)$ & NA & $3.0(1.6-5.5)$ & NA & NA & $\begin{array}{l}3.0 \\
(1.6-5.5)\end{array}$ & NA & NA & NA \\
\hline
\end{tabular}

$\mathrm{CI}$ = confidence interval; ISUP = International Society of Urological Pathology; MRI = magnetic resonance imaging; MRI pathway = magnetic resonance imaging with or without magnetic resonance imaging-targeted biopsy; $\mathrm{NA}=$ not applicable; $\mathrm{SBx}=$ systematic biopsy.

a Proportion of participants with a positive or negative magnetic resonance imaging result, based on the studies reporting grade 2 or higher.

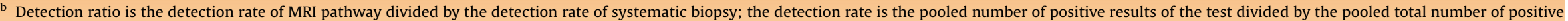
results from both tests.

c Evaluating the difference in detection ratios between the populations (biopsy-naive men vs prior-negative biopsy) for each target condition.

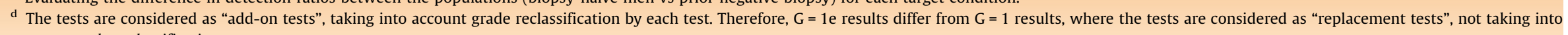
account grade reclassification. 


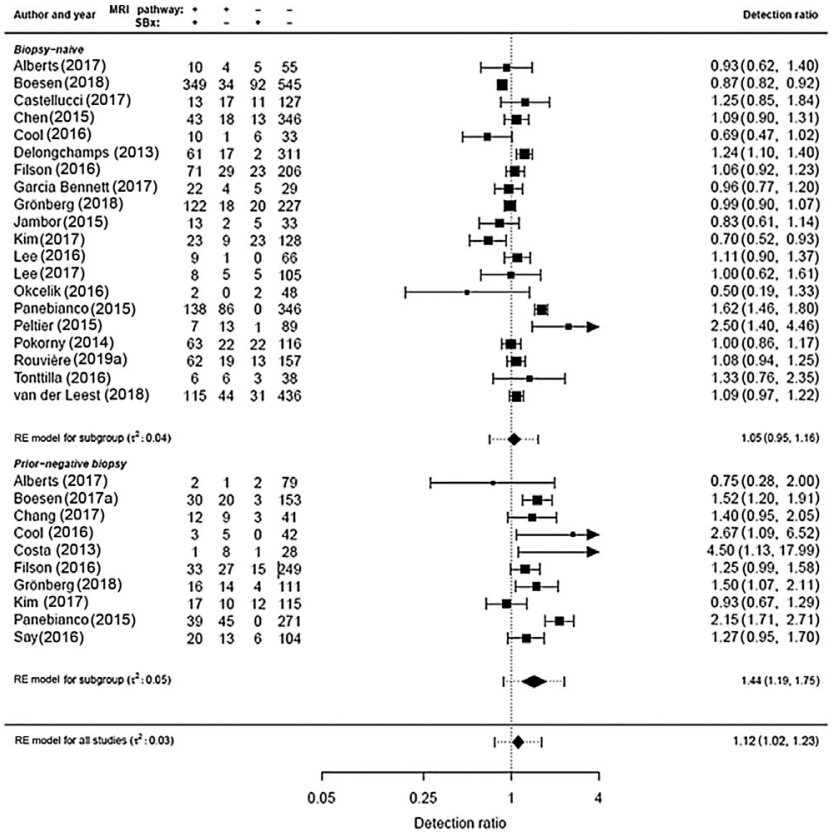

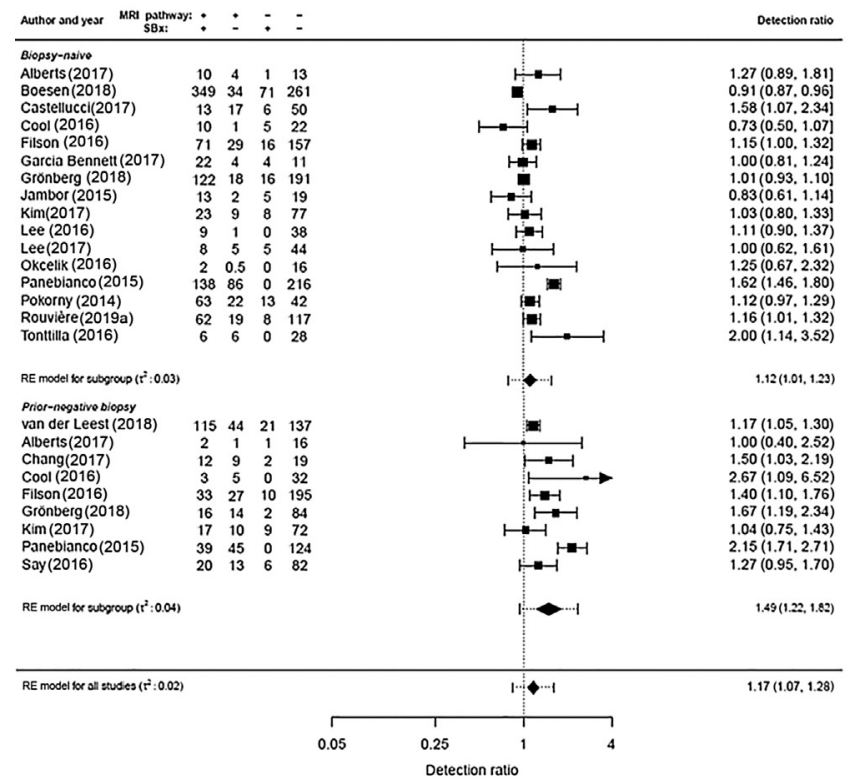

The upper plot is based on all included men; the lower plot is based on MRl-positive men MRI pathway: magnetic resonance imaging with subsequent magnetic resonanoe imaging-targeted biopsy; $\mathbf{S B} x$ systematic biopsy; +: positive test result; : $:$ negative test result; detection ratio: detection rate MRI pathway divided by detection rate SBX; detection rate: pooled number of positive results of one test divided by the pooled total number of positive results from both tests; RE model: random effects model; $\mathbf{v}^{2}$ : Tau 2 (heterogeneity). The continuous lines and brackets indicate study individual $95 \%$ confideno intervals; diamonds indicate the pooled summary estimate $95 \%$ confidenoe intervals; the dashed lines indicate the pooled $95 \%$ predicition intervals.

Fig. 5 - Forest plots of the agreement analysis (MRI pathway vs systematic biopsy) for detecting grade 2 and higher prostate cancer. The upper plot is based on all included men; the lower plot is based on MRI-positive men. Continuous lines and brackets indicate study individual 95\% confidence intervals; diamonds indicate the pooled summary estimate $95 \%$ confidence intervals; and dashed lines indicate the pooled 95\% prediction intervals. Detection rate $=$ pooled number of positive results of one test divided by the pooled total number of positive results from both tests; detection ratio = detection rate of the MRI pathway divided by detection rate of SBx; MRI magnetic resonance imaging; MRI pathway: MRI with subsequent MRI-targeted biopsy; RE model = random-effect model; SBX = systematic biopsy; $\tau^{2}$ : tau-square (heterogeneity); + = positive result; $-=$ negative result. be counselled to pursue clinical and biochemical monitoring as a reasonable alternative for systematic biopsy, as also argued by others [25-27].

Men with positive MRI have a clear indication for MRItargeted biopsy and can opt for additional systematic biopsy. The added value of performing systematic biopsy in MRI-positive men for the detection of grade 2 or higher prostate cancer, however, could be considered as limited with regard to total detection and additional harms. The conditions under which systematic biopsy could be safely avoided in men with positive MRI remain to be defined $[26,28,29]$. When in this population, the MRI pathway does not detect significant prostate cancer, a monitoring approach could be introduced (instead of systematic biopsy), based on clinical, biochemical, and imaging parameters, and would result in a "safety net". This safety net could easily be adopted in the shared decision making in current diagnostic work-up, as already recommended in international guidelines [30-32].

\subsubsection{Strength and weaknesses}

For the in-depth analysis of quantity and quality of evidence, strengths and weaknesses of included studies, and strengths and weaknesses of the review process, we refer to the original Cochrane review [13].

\subsubsection{Context of other research}

Distinguishing between biopsy-naive men and men with a prior-negative biopsy is paramount in daily practice. The agreement analysis, balancing the results of detecting grade 2 or higher prostate cancer, grade 1 prostate cancer, and reduction of biopsies in MRI-negative men, can be compared with selected high-quality studies (Supplementary Table 1). Recently, two multicentre randomised controlled trials in biopsy-naive men $[2,33]$ investigated the MRI pathway and systematic biopsy. Furthermore, two large high-quality prospective multicentre cohort studies $[24,34]$ investigated the agreement of prostate cancer detection between the MRI pathway and systematic biopsy.

The most remarkable differences are the following. Both randomised controlled trials showed that the MRI pathway detected significantly more grade 2 or higher prostate cancer than systematic biopsy [2,33], in contrast to the results from the agreement analyses in this review [13], including the two cohort studies [24,34]. Hence, while the randomised controlled trials showed superiority of the MRI pathway over systematic biopsy, the agreement studies did not. Despite these inconsistencies, none of the studies showed the MRI pathway to be inferior to systematic biopsy in detecting grade 2 or higher prostate cancer. In addition, in this Cochrane review, the proportion of men with grade 2 or higher prostate cancer detected by the MRI pathway was 23.4\% (95\% CI: 19.3-28.1\%), while this was substantially higher in the two randomised controlled trials (Supplementary Table 1). Regarding the proportions of men with grade 1 prostate cancer, the MRI pathway in this review 
detected $14 \%$ (95\% CI: 11-17\%), while this was lower in the two randomised controlled trials. Explanatory reasons might be multiple and are discussed within the context of this review (Cochrane review [13]).

\subsubsection{Future research and perspectives}

Quality control in the MRI pathway should be further employed to improve MRI acquisition, MRI reading, and MRI-targeted biopsy methods. The role of biparametric MRI as well as the different approaches for targeted biopsy (fusion, cognitive/visual, in bore), the route (transrectal/ transperineal), and the clinical validity and utility of artificial intelligence with machine learning tools should be further investigated. Education, training, procedural standardisation, better imaging, and biopsy equipment require a multidisciplinary approach in the management of men with suspected prostate cancer [7,15,35,36]. This diagnostic chain is only as strong as its weakest link [37]. To improve the clinical utility of MRI-driven tests, factors influencing the outcome of the MRI pathway (such as per-lesion instead of a per-patient analysis, number of MRI-targeted biopsy cores, MRI positivity threshold in relation to clinical risk profiles, underlying MRI reading problems, and inaccurate MRI-targeted biopsy) should be further investigated. Risk calculators may aid in balancing harms and benefits by further refining the selection of those men who are at a risk of potentially life-threatening disease. Research should be initiated with recently introduced multivariable risk prediction models, including the MRI suspicion score as an extra input variable, to better identify those who would benefit from MRI and subsequent MRItargeted biopsy, or an additional systematic biopsy, or both [38-42].

\section{Conclusions}

Balancing the potential benefits (reduction of biopsies and a decrease of grade 1 prostate cancer overdiagnosis) against the potential disadvantages (missing some grade 2 or higher prostate cancer), in disregard to further economic metrics (availability and costs), we conclude that the MRI pathway may represent a more favourable diagnostic test than systematic biopsy in all men suspected to have clinically significant prostate cancer. Therefore, performing prostate MRI before any biopsy should be structurally incorporated in the diagnostic work-up. Our certainty in our findings was reduced by study limitations. Furthermore, the MRI pathway relies on experience and skills in acquiring and reading MRI images, on targeting biopsy, and on high-end equipment of MRI and biopsy hardware and software, which are not yet widely available. Based on these considerations, further improvement of the prostate cancer diagnostic pathways should be pursued.

Author contributions: Ivo G. Schoots had full access to all the data in the study and takes responsibility for the integrity of the data and the accuracy of the data analysis.

Study concept and design: Drost, Roobol, Schoots.
Acquisition of data: Drost, Osses.

Analysis and interpretation of data: Drost, Osses, Nieboer, Roobol, Schoots. Drafting of the manuscript: Drost, Schoots.

Critical revision of the manuscript for important intellectual content: Bangma, Steyerberg, Roobol.

Statistical analysis: Drost, Nieboer.

Obtaining funding: None.

Administrative, technical, or material support: None.

Supervision: Schoots.

Other: None.

Financial disclosures: Ivo G. Schoots certifies that all conflicts of interest, including specific financial interests and relationships and affiliations relevant to the subject matter or materials discussed in the manuscript (eg, employment/affiliation, grants or funding, consultancies, honoraria, stock ownership or options, expert testimony, royalties, or patents filed, received, or pending), are the following: Frank-Jan H. Drost, Daniel F. Osses, and Daan Nieboer: none known. Ewout W. Steyerberg reports the following relevant financial activities outside the submitted work: receives royalties from Springer for the textbook entitled Clinical prediction models. Chris H Bangma and Monique J Roobol: none known. Ivo G. Schoots reports the following relevant activities related to the submitted work: a guideline associate panel member of the EAU-ESTROESUR-SIOG Guidelines on Prostate Cancer.

\section{Funding/Support and role of the sponsor: None.}

Acknowledgements: We thank Mr. Wichor M. Bramer, Information Specialist, Medical Library, Erasmus University Medical Centre, Rotterdam, for conducting the systematic literature search. We thank Myriam M.G. Hunink for critically evaluating the protocol. We also thank Jan Verbeek for his thoughts and input in discussions. We thank Caroline M. Moore, Anwar R. Padhani, and Olivier Rouviere for their extensive review. We wish to acknowledge the support of the Cochrane Collaboration's Diagnostic Test Accuracy editorial team, the Cochrane Urology editorial team, and the peer referees for their assistance. We thank Philipp Dahm as the Coordinating Editor of Cochrane Urology and a member of the US GRADE Network for his assistance in generating the GRADE summary of findings tables.

\section{Appendix A. Supplementary data}

Supplementary data associated with this article can be found, in the online version, at https://doi.org/10.1016/j. eururo.2019.06.023.

\section{References}

[1] Alberts AR, Schoots IG, Roobol MJ. Prostate-specific antigen-based prostate cancer screening: past and future. Int J Urol 2015;22:524-32.

[2] Kasivisvanathan V, Rannikko AS, Borghi M, et al. MRI-targeted or standard biopsy for prostate-cancer diagnosis. N Engl J Med 2018;378:1767-77.

[3] De Rooij M, Hamoen EH, Futterer JJ, Barentsz JO, Rovers MM. Accuracy of multiparametric MRI for prostate cancer detection: a meta-analysis. Am J Roentgenol 2014;202:343-51.

[4] Futterer JJ, Briganti A, De Visschere P, et al. Can clinically significant prostate cancer be detected with multiparametric magnetic resonance imaging? A systematic review of the literature. Eur Urol 2015;68:1045-53.

[5] Gayet M, Van der Aa A, Beerlage HP, Schrier BP, Mulders PF, Wijkstra $\mathrm{H}$. The value of magnetic resonance imaging and ultrasonography (MRI/US)-fusion biopsy platforms in prostate cancer detection: a systematic review. BJU Int 2016;117:392-400. 
[6] Hamoen EH, de Rooij M, Witjes JA, Barentsz JO, Rovers MM. Use of the Prostate Imaging Reporting and Data System (PI-RADS) for prostate cancer detection with multiparametric magnetic resonance imaging: a diagnostic meta-analysis. Eur Urol 2015;67:1112-21.

[7] Moore CM, Robertson NL, Arsanious N, et al. Image-guided prostate biopsy using magnetic resonance imaging-derived targets: a systematic review. Eur Urol 2013;63:125-40.

[8] Schoots IG, Roobol MJ, Nieboer D, Bangma CH, Steyerberg EW, Hunink MG. Magnetic resonance imaging-targeted biopsy may enhance the diagnostic accuracy of significant prostate cancer detection compared to standard transrectal ultrasound-guided biopsy: a systematic review and meta-analysis. Eur Urol 2015;68: 438-50.

[9] Valerio M, Donaldson I, Emberton M, et al. Detection of clinically significant prostate cancer using magnetic resonance imaging-ultrasound fusion targeted biopsy: a systematic review. Eur Urol 2015;68:8-19.

[10] Van Hove A, Savoie PH, Maurin C, et al. Comparison of image-guided targeted biopsies versus systematic randomized biopsies in the detection of prostate cancer: a systematic literature review of well-designed studies. World J Urol 2014;32:847-58.

[11] Woo S, Suh CH, Kim SY, Cho JY, Kim SH, Moon MH. Head-to-head comparison between biparametric and multiparametric MRI for the diagnosis of prostate cancer: a systematic review and meta-analysis. AJR Am J Roentgenol 2018;211:W226-41.

[12] Robertson NL, Hu Y, Ahmed HU, Freeman A, Barratt D, Emberton M. Prostate cancer risk inflation as a consequence of image-targeted biopsy of the prostate: a computer simulation study. Eur Urol 2014;65:628-34.

[13] Drost FH, Osses DF, Nieboer D, et al. Prostate MRI, with or without MRI-targeted biopsy, and systematic biopsy for detecting prostate cancer. Cochrane Database Syst Rev 2019;4:Cd012663.

[14] Dickinson L, Ahmed HU, Allen C, et al. Magnetic resonance imaging for the detection, localisation, and characterisation of prostate cancer: recommendations from a European consensus meeting. Eur Urol 2011;59:477-94.

[15] Weinreb JC, Barentsz JO, Choyke PL, et al. PI-RADS Prostate ImagingReporting and Data System: 2015, version 2. Eur Urol 2016;69:16-40.

[16] Kuru TH, Wadhwa K, Chang RT, et al. Definitions of terms, processes and a minimum dataset for transperineal prostate biopsies: a standardization approach of the Ginsburg for Enhanced Prostate Diagnostics. BJU Int 2013;112:568-77.

[17] Sivaraman A, Sanchez-Salas R, Barret E, et al. Transperineal templateguided mapping biopsy of the prostate. Int J Urol 2015;22:146-51.

[18] Moore CM, Kasivisvanathan V, Eggener S, et al.Standards of reporting for MRI-targeted biopsy studies (START) of the prostate: recommendations from an International Working Group. Eur Urol 2013;64:544-52.

[19] Epstein JI, Egevad L, Amin MB, et al. The 2014 International Society of Urological Pathology (ISUP) consensus conference on Gleason grading of prostatic carcinoma: definition of grading patterns and proposal for a new grading system. Am J Surg Pathol 2016;40:244-52.

[20] Whiting PF, Rutjes AW, Westwood ME, et al. QUADAS-2: a revised tool for the quality assessment of diagnostic accuracy studies. Ann Intern Med 2011;155:529-36.

[21] Macaskill P, Gatsonis C, Deeks JJ, Harbord RM, Takwoingi Y. Chapter 10: analysing and presenting results. In: Deeks JJ, Bossuyt PM, Gatsonis C, editors. Cochrane handbook for systematic reviews of diagnostic test accuracy version 1.0. The Cochrane Collaboration; 2010,, srdta.cochrane.org/.

[22] Schünemann HJ, Oxman AD, Brozek J, et al. Grading quality of evidence and strength of recommendations for diagnostic tests and strategies. Br Med J 2008;336:1106-10.

[23] Barentsz JO, Richenberg J, Clements R, et al. ESUR prostate MR guidelines 2012. Eur Radiol 2012;22:746-57.
[24] van der Leest M, Cornel E, Israel B, et al. Head-to-head comparison of transrectal ultrasound-guided prostate biopsy versus multiparametric prostate resonance imaging with subsequent magnetic resonance-guided biopsy in biopsy-naive men with elevated prostate-specific antigen: a large prospective multicenter clinical study. Eur Urol 2019;75:570-8.

[25] Moldovan PC, Van den Broeck T, Sylvester R, et al. What is the negative predictive value of multiparametric magnetic resonance imaging in excluding prostate cancer at biopsy? A systematic review and meta-analysis from the European Association of Urology Prostate Cancer Guidelines Panel. Eur Urol 2017;72:250-66.

[26] Padhani AR, Weinreb J, Rosenkrantz AB, Villeirs G, Turkbey B, Barentsz J. Prostate Imaging-Reporting and Data System Steering Committee: PI-RADS v2 status update and future directions. Eur Urol 2019;75:358-96.

[27] Panebianco V, Barchetti G, Simone G, et al. Negative multiparametric magnetic resonance imaging for prostate cancer: what's next? Eur Urol 2018;74:48-54.

[28] Richenberg J, Logager V, Panebianco V, Rouviere O, Villeirs G, Schoots IG. The primacy of multiparametric MRI in men with suspected prostate cancer. In press. https://doi.org/10.1007/ s00330-019-06166-z.

[29] Rouvière O, Souchon R, Melodelima C. Pitfalls in interpreting positive and negative predictive values: application to prostate multiparametric magnetic resonance imaging. Diagn Interv Imaging 2018;99:515-8.

[30] Carter HB, Albertsen PC, Barry MJ, et al. Early detection of prostate cancer: AUA guideline. http://www.auanet.org/guidelines/ prostate-cancer-early-detection-(2013-reviewed-for-currency2018)-x26372018.

[31] Mottet N, Van den Bergh RCN, Briers E, et al. European Association of Urology: guideline on prostate cancer. 2018 In: https://uroweb. org/guideline/prostate-cancer/

[32] National Comprehensive Cancer Network (NCCN). Guidelines on prostate cancer: 2018, update. 2018 In: http://www.nccn.org/ professionals/physician_gls/default.aspx

[33] Porpiglia F, Manfredi M, Mele F, et al. Diagnostic pathway with multiparametric magnetic resonance imaging versus standard pathway: results from a randomized prospective study in biopsy-naive patients with suspected prostate cancer. Eur Urol 2017;72: 282-8.

[34] Rouvière O, Puech P, Renard-Penna R, et al. Use of prostate systematic and targeted biopsy on the basis of multiparametric MRI in biopsy-naive patients (MRI-FIRST): a prospective, multicentre, paired diagnostic study. Lancet Oncol 2019;20:100-9.

[35] Moore CM, Giganti F, Albertsen P, et al. Reporting magnetic resonance imaging in men on active surveillance for prostate cancer: the precise recommendations-a report of a European School of Oncology task force. Eur Urol 2017;71:648-55.

[36] Puech P, Randazzo M, Ouzzane A, et al. How are we going to train a generation of radiologists (and urologists) to read prostate MRI? Curr Opin Urol 2015;25:522-35.

[37] Rouvière O, Schoots IG, Mottet N, EAU-EANM-ESTRO-ESUR-SIOG Prostate Cancer Guidelines Panel. Multiparametric magnetic resonance imaging before prostate biopsy: a chain is only as strong as its weakest link. Eur Urol 2019;75:889-90.

[38] Alberts AR, Roobol MJ, Verbeek JF, et al. Prediction of high-grade prostate cancer following multiparametric magnetic resonance imaging: improving the Rotterdam European Randomized Study of Screening for Prostate Cancer risk calculators. Eur Urol 2019;75: 310-8.

[39] Ankerst DP, Straubinger J, Selig K, et al. A contemporary prostate biopsy risk calculator based on multiple heterogeneous cohorts. Eur Urol 2018;74:197-203.

[40] Foley RW, Maweni RM, Gorman L, et al. European Randomised Study of Screening for Prostate Cancer (ERSPC) risk calculators significantly outperform the Prostate Cancer Prevention Trial 
(PCPT) 2.0 in the prediction of prostate cancer: a multi-institutional study. BJU Int 2016;118:706-13.

[41] Mehralivand S, Shih JH, Rais-Bahrami S, et al. A magnetic resonance imaging-based prediction model for prostate biopsy risk stratification. JAMA Oncol 2018;4:678-85.
[42] Radtke JP, Wiesenfarth M, Kesch C, et al. Combined clinical parameters and multiparametric magnetic resonance imaging for advanced risk modeling of prostate cancer-patient-tailored risk stratification can reduce unnecessary biopsies. Eur Urol 2017;72: 888-96. 